\title{
Transcranial direct current stimulation with functional magnetic resonance imaging: A detailed validation and operational guide
}

\author{
Davide Nardo*1,2, Megan Creasey*3, Clive Negus ${ }^{3}$, Katerina Pappa ${ }^{1,4}$, Alphonso Reid $^{3}$, Oliver \\ Josephs $^{3}$, Martina F. Callaghan ${ }^{3}$, Jenny T. Crinion ${ }^{1,3}$ \\ ${ }^{1}$ Institute of Cognitive Neuroscience, University College London, UK. \\ ${ }^{2}$ MRC Cognition and Brain Sciences Unit, University of Cambridge, UK. \\ ${ }^{3}$ Wellcome Centre for Human Neuroimaging, UCL Queen Square Institute of Neurology, University College \\ London, UK. \\ ${ }^{4}$ Institute of Health \& Wellbeing, University of Glasgow, UK
}

[* Authors contributed equally]

\section{Abstract}

Introduction: Transcranial direct current stimulation (tDCS) is a non-invasive brain stimulation technique used to modulate human brain and behavioural function in both research and clinical interventions. The combination of functional magnetic resonance imaging (fMRI) with tDCS enables researchers to directly test causal contributions of stimulated brain regions, answering questions about the physiology and neural mechanisms underlying behaviour. Despite the promise of the technique, advances have been hampered by technical challenges and methodological variability between studies, confounding comparability/replicability.

Methods: Here tDCS-fMRI at 3T was developed for a series of experiments investigating language recovery after stroke. To validate the method one healthy volunteer completed an fMRI paradigm with three conditions (i) no-tDCS, (ii) Sham-tDCS, (iii) $2 \mathrm{~mA}$ Anodal-tDCS. MR data were analysed in SPM12 with region of interest (ROI) analyses of the two electrode and reference sites.

Results: Quality assessment indicated no visible signal dropouts or distortions introduced by the tDCS equipment. After modelling scanner drift, motion-related variance, and temporal autocorrelation we found no field inhomogeneity in functional sensitivity metrics across conditions in grey matter and in the three ROIs.

Discussion: Key safety factors and risk mitigation strategies that must be taken into consideration when integrating tDCS into an fMRI environment are outlined. To obtain reliable results, we provide practical solutions to technical challenges and complications of the method. It is hoped that sharing these data and SOP will promote methodological replication in future studies, enhancing the quality of tDCS-fMRI application, and improve the reliability of scientific results in this field.

Conclusion: The method and data provided here provide a technically safe, reliable tDCSfMRI procedure to obtain high quality MR data. The detailed framework of the SOP (in extended data) systematically reports the technical and procedural elements of our tDCS-fMRI approach, which we hope can be adopted and prove useful in future studies.

Abstract word count: 300 


\section{Keywords}

tDCS - transcranial direct current stimulation; tES - transcranial electrical brain stimulation; fMRI - functional magnetic resonance imaging; SOP - standard operating procedure; safety factors; technical challenges; implementation guide.

Corresponding author: Megan Creasey (m.creasey@ucl.ac.uk).

Author roles: Nardo, D: Investigation, Methodology, Visualisation, Validation, Project Administration, Writing - Original Draft Preparation and Review and Editing. Creasey, M: Visualisation, Writing - Review and Editing. Negus, C: Methodology, Visualisation, Writing Review and Editing. Pappa, K: Investigation, Methodology, Project Administration, Writing Original Draft Preparation. Reid, A: Resources, Software. Josephs, O: Methodology, Validation. Callaghan, MF: Methodology, Supervision, Visualisation, Writing - Review and Editing. Crinion, J: Conceptualisation, Investigation, Methodology, Supervision, Visualisation, Validation, Writing - Review and Editing.

Grant information: This study was supported by Wellcome funding (106161/Z/14/Z to JC) (203147/Z/16/Z to WCHN). The funders had no participation in the design and results of this study.

Data Availability: Data are provided as Extended Data to this article.

Competing interests: No competing interests were disclosed.

Acknowledgments: Bradbury, $\mathrm{D}^{3}$. Laboratory manager - contributed to reviewing safety documentation, risk assessments, and smooth implementation of the SOP. Schellhorn, K. from neuroConn for his technical assistance and guidance in setting up 2mA tDCS-fMRI at WCHN.

Article word count: 4538 (including Figures) 


\section{Introduction}

Transcranial direct current stimulation (tDCS) is one method of non-invasive transcranial electrical brain stimulation (tES). The technique uses a small current (1-2mA) applied via scalp electrodes for up to 20 minutes in human volunteers. During tDCS stimulation current flows between the surface electrodes - passing through the brain to complete a circuit. Increasing interest in the technique has stemmed from a desire to explore and alter the physiological mechanisms underlying basic human motor, perceptual and cognitive processes. Its immediate and long-lasting effects, albeit with unpredictable cognitive results (Thair et al., 2017), its safety and tolerability (Antal et al., 2017; Bikson et al., 2016), non-complex technical requirements, and low cost (Woods et al., 2016) have made it an attractive treatment option for several neurological and psychiatric disorders (Brunoni et al., 2019; Schulz et al., 2013). However, the neural mechanisms by which tDCS modulates human brain and behavioural function are still unclear. An increased understanding of these mechanisms would allow more effective and individualised targeted interventions to be developed.

With the advent of magnetic resonance imaging (MRI)-compatible tES devices, concurrent tDCS and functional magnetic resonance imaging (fMRI) is technically feasible. Using the "perturb and measure" approach (Paus, 2005) the casual contributions of a stimulated brain region's function can be directly assessed online, during (cognitive) task performance, offering researchers a unique opportunity to answer basic questions about underlying physiology. Combined with the high spatial resolution that fMRI offers across the entire brain, research has shown that tDCS effects are not spatially restricted to the brain region directly underneath the stimulating electrode. Indeed, tDCS affects multiple regions due in part to distributed current flow and brain connectivity (Abellaneda-Pérez et al., 2020; Mondino et al., 2020), including anatomically distant but functionally connected regions (Chib et al., 2013; Stagg et al., 2013). This has resulted in a number of important guides published on the technique (Meinzer et al., 2014; Woods et al., 2016; Thair et al., 2017). A number of additional studies have illustrated that the technique can be conducted safely (e.g., minimising risk of local electrode heating and skin burning) without posing severe data quality constraints as long as proper procedures are followed (Antal et al., 2014; Esmaeilpour et al., 2020). Nonetheless, advances in the field of tDCS-fMRI have been hampered by the methodological variability between studies, which limits comparisons between studies and replicability of findings (Esmaeilpour et al., 2020).

Surprisingly, despite an increasing number of research labs using tES and fMRI, a recent systematic review of 222 tES-fMRI experiments (181 tDCS) published before February 1, 2019 , found there were no two studies with the same methodological parameters to replicate findings (Ghobadi-Azbari et al., 2020). The authors conclude that, because the methodology progressed largely independently between different research groups, it resulted in diverse protocols and findings across research groups. Importantly, the heterogeneous mixture of findings, cannot always be interpreted independently from the methodological parameters (Nitsche et al., 2015). Indeed, concurrent tES-fMRI studies are more susceptible to artefactual noise than other fMRI scenarios, and may risk false positive BOLD (Blood Oxygen Level Dependent) signal results (Antal et al., 2014; Woods et al., 2016). Very few studies have 
provided data on change in the magnetic field in relation to concurrent tDCS-fMRI, the magnitude and nature of which are likely to depend on the exact experimental setup within each lab, for each fMRI paradigm. This highlights the need for careful consideration of tDCSfMRI results and how the lack of methodological overlap between studies to date makes any meta-analysis and/or conclusion about mechanistic effects of tDCS extremely challenging.

To address the inconsistency of methodological approaches used in tDCS-fMRI studies, here we provide a step-by-step guide through the Standard Operating Procedure (SOP) governing safe operation of tDCS-fMRI at the Wellcome Centre for Human Neuroimaging (WCHN). The SOP was designed to provide sufficiently detailed methodological information so that methods can be precisely replicated. It was developed for a series of experiments investigating language recovery after stroke but can be adapted for any study which uses fMRI to investigate the mechanisms underlying tDCS effects.

The SOP focuses on methods required for the safe use of tDCS equipment in the MRI environment, whilst maintaining high image quality to obtain reliable results. It is followed by a discussion of the key risk factors (safety and image artefact) associated with concurrent tDCS-fMRI and risk mitigation strategies implemented in our Lab. By sharing this information, we aim to aid replication of methodological approaches across studies and sites and increase replicability of published evidence in the field.

\section{Method}

\section{Study Details}

This SOP was originally designed for an fMRI-tDCS study conducted with healthy older control participants. This was followed by a series of studies exploring a clinical population of patients with aphasia following stroke. The fMRI-tDCS procedure has been found to be well tolerated by healthy older adults (Holland et al., 2011) and patients with aphasia (Ondobaka et al., 2020) and at the time of writing, we have collected data from over 36 stroke patients with no reported adverse events or tolerance issues. In both healthy and stroke cases, participants were not able to reliably detect differences between the sham and stimulation conditions. While these studies focussed on language production, requiring specific additional equipment such as headphones and microphone, what follows is a detailed SOP that could be adapted for other experimental paradigms.

\section{tDCS Equipment and Montage}

We used MR-compatible tDCS equipment (NeuroConn DC-stimulator; https://www.neurocaregroup.com/dc_stimulator_mr.html) to apply $2 \mathrm{~mA}$ anodal tDCS delivered for 20 mins to the frontal cortex. In the Extended Data, we report step by step the detailed procedure (the SOP) we have put in place. In what follows we report a summary. Besides MRI safety contraindications, participants had to be screened for additional tDCSspecific contraindications. tDCS equipment had to be put in place in three different environments (Control Room, Scan Room, and Testing Room). EEG conductive paste (Ten20) 
was used as the electrode contact medium, and 3M Coban elastic wrap bandage was used secure electrode placement. The tDCS stimulator was placed inside a radiofrequency (RF)-shielded box during the experiment, to minimise any RF interference between the Scan Room and the external environment, and the stimulation was triggered by the scanner via a Fibre-Optic. A tailor-made foam-base was created and use to facilitate set-up in the scanner bore, and guarantee consistency across sessions. Our procedure envisages three people (typically two researchers and one radiographer), whose roles and responsibilities are defined in detail. Participants were suitably prepared before coming to the scanner, including MR-safety checks and two impedance checks (one in the Testing Room, another in the Scan Room). Participants were connected to the tDCS equipment by making sure that no loops were created in the scanner, and loops were avoided along the whole length of the various cables in the Scan Room. A detailed procedure to safely remove participants from the scanner/tDCS equipment in case of emergency is also provided in the Extended Data.

\section{Quality assessment of MR images}

In order to compare the functional sensitivity of the three stimulation conditions (i.e., No-tDCS setup in place, Sham-tDCS, and Anodal-tDCS), we computed the t-score testing for the mean signal (cf. Corbin et al., 2018). In the simple case of the design matrix being a unitary vector, with length corresponding to the number of temporal samples, and there being no temporal correlation in the data, this metric reduces to the commonly used temporal signal-to-noise ratio (tSNR) weighted by the square root of the number of samples. We computed this functional sensitivity metric both at the whole-brain level, restricted to grey matter tissue, and within three regions-of-interest (ROIs) located beneath the anodal electrode, the cathodal electrode, and an independent site remote from the electrodes, which was used as a reference.

\section{fMRI acquisition}

MR data were collected from a healthy participant (F, 46) on a 3T Siemens PrismaFit system (Siemens, Erlangen, Germany) at the WCHN. Data included a T1-weighted MPRAGE acquisition for anatomical reference $(\mathrm{TR}=2.53 \mathrm{~s}, \mathrm{TE}=3.34 \mathrm{~ms}$, voxel size $=1 \times 1 \times 1 \mathrm{~mm}$, field of view $=256 \times 256 \times 176 \mathrm{~mm}^{3}$ ), dual gradient-echo based field maps to map $\mathrm{B}_{0}$ field inhomogeneity and subsequently apply distortion correction to the functional images, and $\mathrm{T} 2 *_{-}$ weighted echo-planar images (EPI) using a 20-channel head coil and acquired during restingstate, with the following parameters: $\mathrm{TR}=3.36 \mathrm{~s}, \mathrm{TE}=30 \mathrm{~ms}, 48$ axial slices with ascending slice ordering, slice thickness $=2.5 \mathrm{~mm}$, inter-slice gap $=0.5 \mathrm{~mm}$, in-plane resolution $=3 \times 3$ $\mathrm{mm}$, flip-angle $90^{\circ}$. A total of 70 volumes (65 of interest and 5 dummies) were acquired in each of three consecutive runs, lasting approximately 4 min each.

\section{fMRI preprocessing}

Functional data were preprocessed and analysed in native space as defined by (i.e., coregistered with) the anatomical image using Statistical Parametric Mapping software (SPM12; www.fil.ion.ucl.ac.uk/spm) running under Matlab 2020a (MathWorks, Natick, MA). These Matlab scripts are provided in the Extended Data. All functional volumes of interest were realigned and unwarped using session-specific voxel displacement maps derived from the $\mathrm{B}_{0}$ 
mapping data. The structural image was segmented into grey matter, white matter, and cerebrospinal fluid.

\section{fMRI analysis}

Statistical analyses were performed in a run-specific fashion. Parameter estimates were calculated for all brain voxels using the General Linear Model (GLM) as implemented in SPM. To remove any low-frequency drifts, data were high-pass filtered using a set of discrete cosine functions with a cut-off period of 128s. A GLM consisting of the nuisance regressors describing motion (given the experiment was task-free) was evaluated, and the t-score testing for the mean signal extracted (Figure 1).

\section{Regions-of-interest (ROIs)}

ROIs were created in two steps. First, spheres with a $40 \mathrm{~mm}$ radius were created around the cortical projections (using the anatomical image as a reference) of the anodal electrode (corresponding to FC5 in a 10-20 system), cathodal electrode (corresponding to FP2), and an independent site remote from the electrodes as a reference (corresponding to PZ), using the MarsBaR toolbox for SPM (http://marsbar.sourceforge.net). Second, each of these spherical ROIs (in native space) was (inclusively) combined with the segmented grey matter tissue from the T1-weighted image (Figure 2).

\section{Results}

Figure 3 shows the frequency distribution and t-score of the mean values (across rows) extracted from the GM, anode (FC5), cathode (FP2) and reference (PZ) ROIs (across columns). There is a high level of overlap for the t-score of the mean distributions in the Sham- and NotDCS conditions. A shift to higher t-score of the mean values is evident for the Anodal-tDCS case. The frequency distributions overlap in each ROI except the cathode ROI (FP2), where a shift to higher frequency is observed. However, the width of the distribution, reflecting field inhomogeneity, is not increased. 


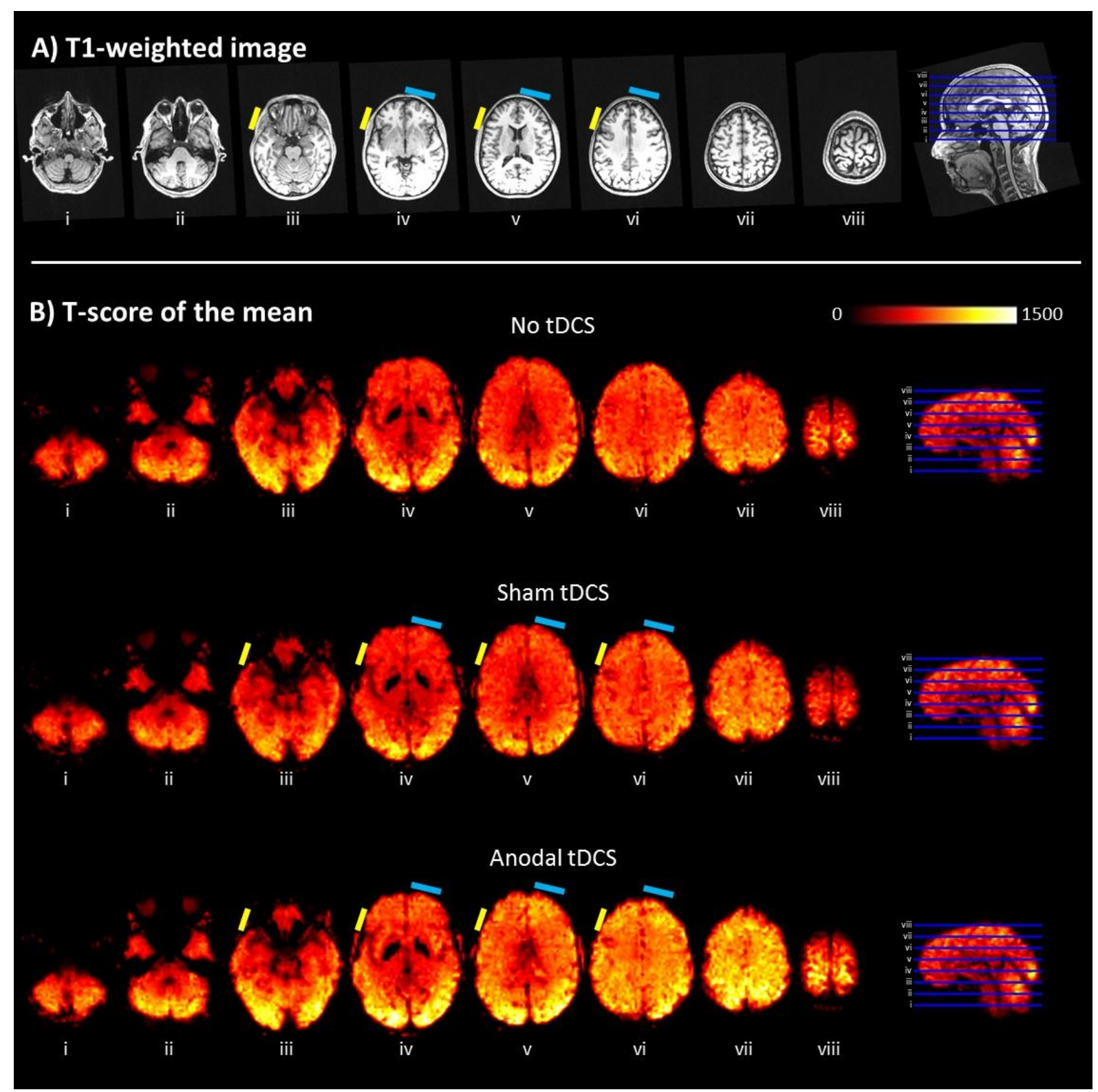

Figure 1 - Quality assessment of MR images acquired on one participant. A) Highresolution ( $1 \mathrm{~mm}$, isotropic) structural T1-weighted image denoting electrode locations shown as a reference. B) t-score of the mean maps. In all panels, axial slices (in ascending order from $i$ to viii with location denoted by the blue lines on the sagittal section on the right) are $15 \mathrm{~mm}$ apart. The approximate position of the electrodes is indicated by the coloured small rectangles ( anodal $=$ yellow; cathodal $=$ light blue $)$. There are no visible signal dropouts or distortions introduced by the electrodes and/or conductive paste. The functional sensitivity measures ( $t$ scores of the mean) are comparable across stimulating conditions, though highest in the Anodal-tDCS case. 


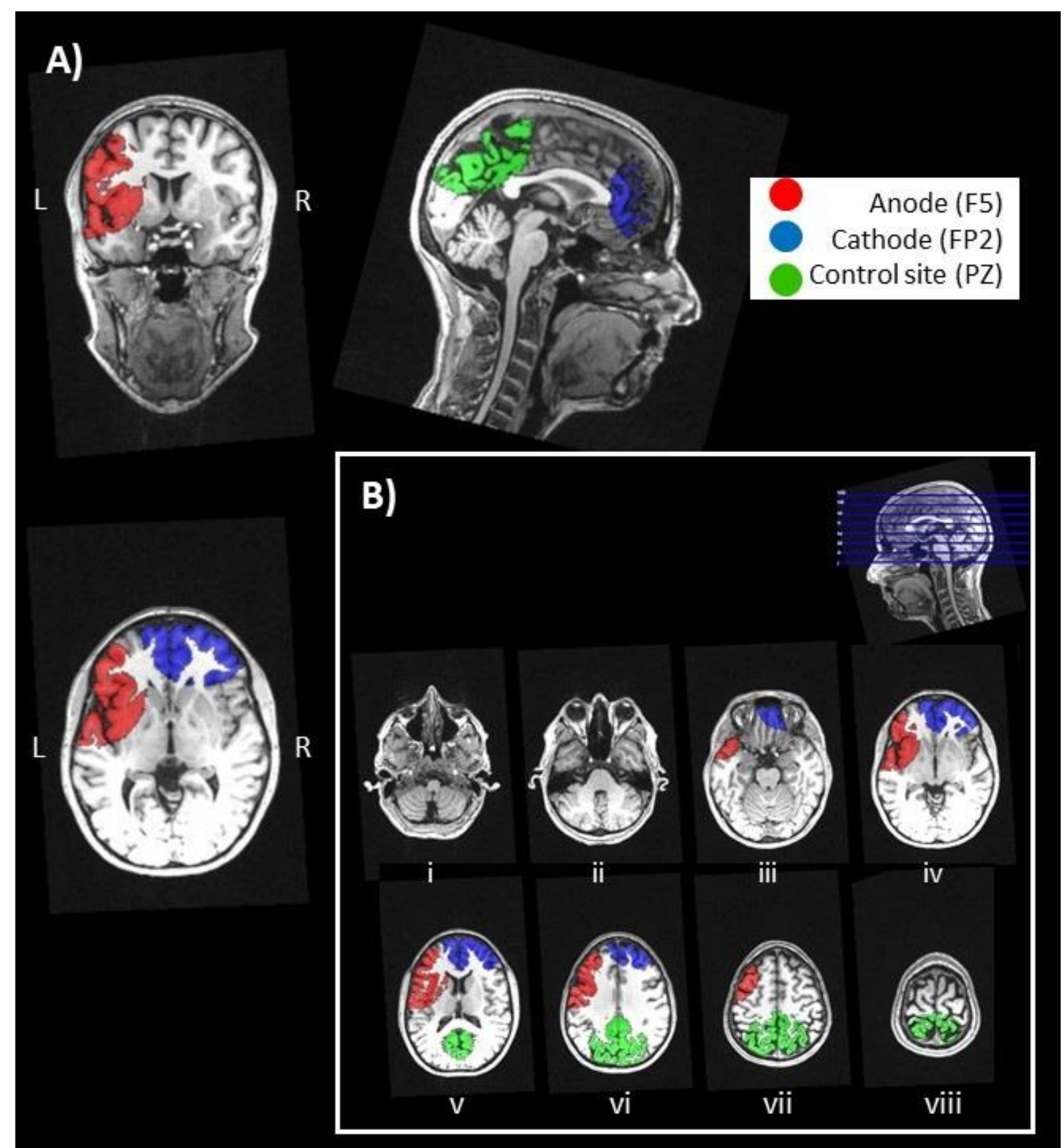

Figure 2 - Regions-of-interest (ROIs). A) Locations of the three ROIs tested overlaid on the coronal, sagittal, and axial sections of a T1-weighted image. ROIs were defined beneath the anodal electrode (in red, corresponding to FC5 in a 10-20 system), cathodal electrode (in blue, corresponding to FP2) and at an independent site remote from the electrodes, used as a reference (in green, corresponding to PZ). B) Locations of the three ROIs tested overlaid on the axial sections shown in Figure 1. 

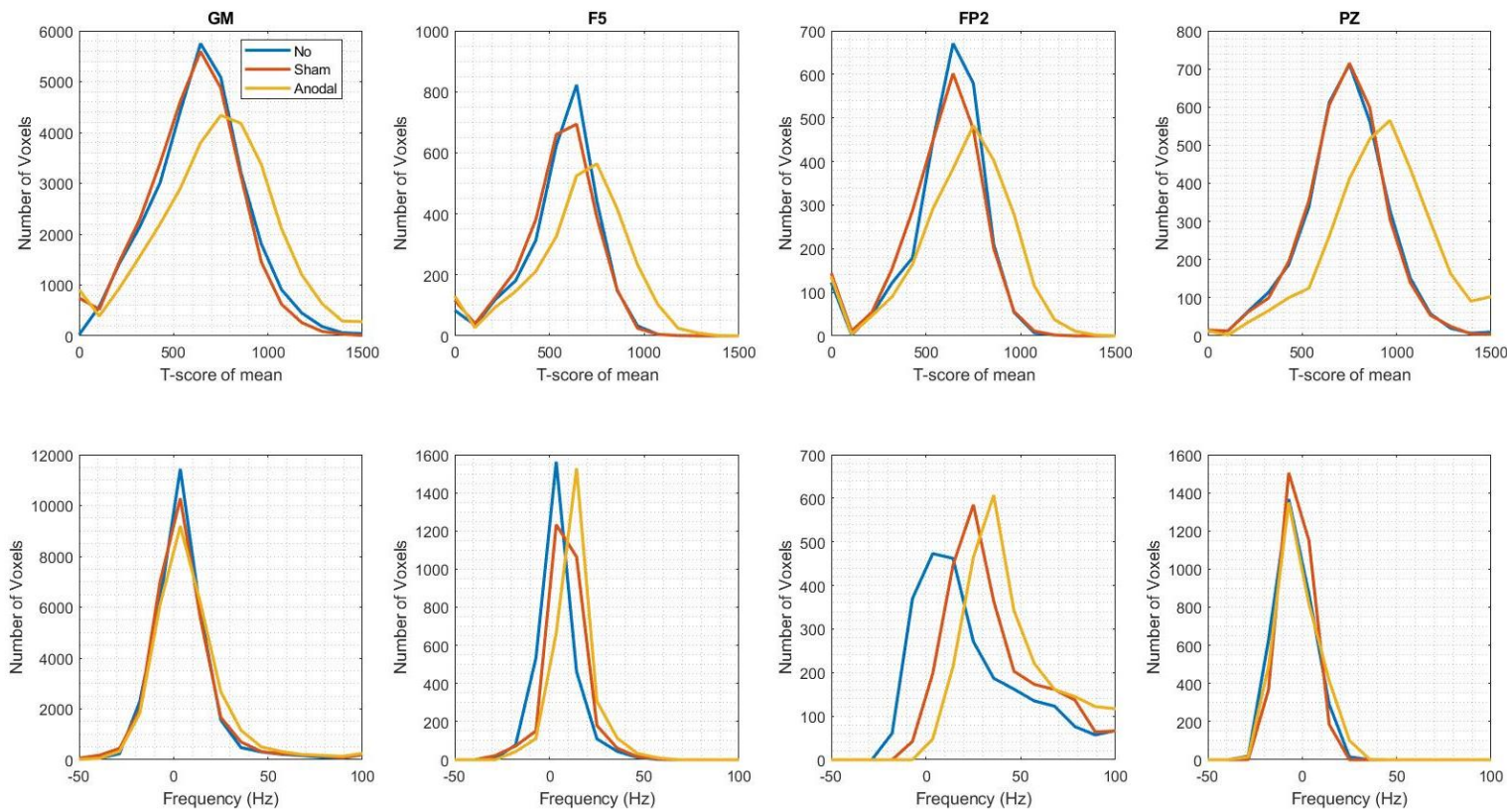

Figure 3 - Functional sensitivity metrics across conditions in grey matter (leftmost column) and in the three ROIs (from left to right: anodal electrode - FC5, cathodal electrode - FP2, independent site remote from the electrodes $-\mathrm{PZ}$ ). Top row: $t$-score of the mean. This measure is extracted from the GLM used for the functional analyses after modelling scanner drift, motion-related variance, and temporal autocorrelation. The t-scores of the mean values with the tDCS equipment in place (i.e., Anodal and Sham conditions) are comparable, if not higher than the No-tDCS condition (i.e., functional sensitivity was not degraded). Bottom row: Offset frequency (measured in $\mathrm{Hz}$ ) to capture field inhomogeneity. The substantial overlap among the three distributions shows that no field inhomogeneity is introduced by the tDCS equipment. Global offsets are observed between conditions in the cathodal (FP2) ROI, but the distribution is in fact broader, indicative of poorer field homogeneity, in the No-tDCS condition.

\section{Discussion}

Combining non-invasive neuro-stimulation and functional neuroimaging techniques can provide a unique opportunity to understand the immediate and long-lasting effects of transcranial direct current stimulation (tDCS) on the brain. At the WCHN, tDCS is being used alongside functional magnetic resonance imaging (fMRI) in order to understand the neural mechanisms underlying tDCS behavioural effects. The combination of fMRI and tDCS methods, including simultaneous/concurrent tDCS-fMRI application can provide unique insight into the neuromodulatory effects of tDCS not only in the targeted brain regions, but also their interconnected networks. The ultimate aim of these mechanistic experiments is to find a relationship between behavioural and neural responses to tDCS. Here, we have shared our detailed procedural methodologies with the aim of increasing replicability of tDCS-fMRI methods and reliability of results of future studies. We hope this will in turn enable the field to gain greater insight into the mechanisms underpinning neural and behavioural modulation by tDCS, which would open up new directions within scientific research and clinical applications, such as developing targeted and meaningful therapies. 
In this section, we first focus on discussing the identified safety risks and accompanying risk mitigations that are specific to the incorporation of the tDCS equipment into the MRI environment that were considered critical during the writing of this operational procedure. Then we discuss the specific challenges of concurrent tDCS-fMRI data and how acquisition of appropriate $\mathrm{B}_{0}$ field map data can help allay concerns over artefacts and false positive functional results from perturbation of the magnetic field. To date, this protocol has proved a safe and reliable means of obtaining high quality fMRI data concurrently with the application of $2 \mathrm{~mA}$ anodal tDCS (20 mins) in over 18 healthy older adults and 36 aphasic stroke patients.

\section{Safety considerations for tDCS in the MRI environment}

The MRI environment poses a number of significant risk factors, primarily due to the various comparatively strong magnetic fields used, which can vary in both space and time. The main magnetic field ( 3 Tesla in our case) can exert significant forces on ferrous objects or currentcarrying conductors. The primary risk to be mitigated against is the introduction of any ferrous components. The NeuroConn DC stimulator Outer-Box contains an RF filter with ferrous components. This Outer-Box should never enter the Scan Room to prevent it from becoming a projectile under the force exerted by the main magnetic field. It should be housed within the waveguide and always be placed and removed via the Control Room.

The time-varying magnetic fields used to achieve the excitation process in MRI have associated electric fields (i.e., this is an electromagnetic RF field). These can induce current flow, both in the participant and any equipment, and lead to heating. During scanning, the MRI system continually monitors the transmitted power to ensure it is as expected, and within regulatory limits by modelling the specific absorption rate, i.e. the energy deposition in the participant, in the absence of any equipment. To accommodate the introduction of the tDCS equipment, and ensure it does not invalidate the model, further mitigation strategies are adopted. Care is taken to arrange cables without introducing any closed loops in which current could flow, and the electrode leads are run along the centre of the bore using the bespoke Foam-base. This base has a groove that maximises the safety of the cabling configuration by ensuring it is parallel with the bore, centred within the transmitting RF coil's volume without any loops, and running away from the participant. The tDCS equipment also has multiple RF filters incorporated and a high input impedance to minimise the currents that could flow as a result of any induced voltage, which could also be caused by the rapidly switching imaging gradients. Resistors are incorporated into the leads adjacent to the electrode pads to further limit any possible current flow. The MRI compatible electrodes are made from an electrically conductive rubber. It is possible that circumferential RF currents could be set up directly within these relatively large pads but for the low SAR sequences used heating is negligible, as confirmed by previous experiments (Holland et al., 2011). As a further risk mitigation strategy, in our Lab only low power imaging sequences are used.

The MRI scanner is situated within a Faraday cage, a continuous copper foil on a wooden support structure, designed to be impermeable to RF fields and often referred to as the RF cage. The purpose of this cage is to contain any internally-generated RF sources, e.g. the transmit 
coil, within the Scan Room and to prevent any external sources from the everyday environment, which could reduce image quality, from entering the Scan Room. The RF cage is explicitly grounded at a single point to prevent unintended connections to the building's electrical ground. To preserve this condition, all electrical connections to the cage are made via a so called "penetration panel" and are RF filtered to maintain the intended RF isolation. Most RF filters used for this purpose are formed of capacitors and inductors in which the capacitors are connected to the RF screen of the cage, which is itself connected to ground. This means that the filters provide a pathway to ground. If any equipment entering the Scan Room in this way is connected to the participant, they too become part of a grounded circuit. The tDCS equipment has been designed for stimulation of human participants in the MRI environment according to the International Electrotechnical Commission standards (60601-1 Class 1 [battery powered] with Type BF [Body, Floating, i.e., no possible route to ground] applied part standard). The NeuroConn manufacturer achieves this by using electrically insulated non-grounded filters, which ensure that the participant being scanned is not connected to ground. This is an intrinsically safe arrangement because, even if a fault condition were to develop during scanning, such that the participant was brought into contact with high voltages, no conducting path is available for a dangerous current to flow through the participant to ground via the tDCS apparatus. To maintain this BF safety status, the penetration panel has not been used to integrate the tDCS equipment in our lab.

The other means of penetrating the RF cage is via a waveguide - a long cylindrical tube that will only allow signals above a certain frequency, known as the cut-off frequency, to pass and therefore can be used to exclude RF signals that would otherwise interfere with imaging. This is the approach we have used to integrate the tDCS equipment into the scanner environment. While this approach ensures that the equipment and participant are not connected to ground, it also introduces the risk of violating the RF isolation (a data quality requirement) and allowing RF from the external environment into the Scan Room.

Two filters are used to attenuate current flow: the MR-compatible "Inner-Box" minimises any currents flowing in the section of the electrode lead in the bore, while the incompatible "OuterBox" in the waveguide itself prevents current flow in the outer cabling entering the Scan Room. However, these RF filters have limited performance meaning that a risk of RF interference compromising the imaging remains, particularly if there is an equipment fault. This risk motivated the extension of our RF cage to enclose the entire stimulator within a shielded box attached, via shielded flexible metallic tubing, to the outside of the waveguide. This additional box has a removable shielding lid, which creates a robust RF seal via fingerstrip gaskets (e.g., https://hollandshielding.com/Shielding-gasket-solutions-materials\#Fingerstrips) and a viewing aperture that is small enough not to compromise the RF shield but sufficient for operation and monitoring of the tDCS device. The lid also proves a useful means of ensuring that the researcher can remain blind to the experimental tDCS condition.

The electrical signal used by the trigger input to drive the stimulator is Galvanically isolated from the rest of the circuitry by the manufacturer (cf. manual). When the box is manually triggered, this isolation can be verified by visual inspection since no wires are connected to 
anything that could be grounded. In theory, an electrical cable can normally be connected directly from the controlling computer, as long as the Galvanic isolation is certain. However, in our case such a connection would also have compromised the additional RF screening by providing a path for RF current to flow. Therefore, a Fibre-Optic trigger signal from the Stimulus PC enters the shielded box through a small waveguide and is subsequently converted to an electrical signal, via battery-control, to drive the stimulator.

\section{tDCS and fMRI Image Quality and Safety Control Study}

Prior to any neuroscience experiments, and in particular due to the extension to the RF cage, the tDCS equipment setup should be tested to ensure that the integrity of the RF cage isolation had not been compromised. In our case, this was done by measuring the cross-talk between two adjacent MRI scanners with and without the tDCS equipment in place. These tests confirmed that, with the RF shielding solution employed here, the RF noise level was equivalent regardless of the presence of the tDCS equipment. As with any experimental setup, routine quality assurance should also be employed. All equipment should be regularly inspected for damage and maintained in keeping with manufacturer guidelines.

The introduction of an electrical current into the scanner's magnetic field results in further warping of the magnetic field (i.e., field artefact). This artefact is of critical concern for BOLD fMRI protocols, as it may result in false positive patterns in BOLD signal (Antal et al., 2014; Woods et al., 2016). Online tES-fMRI studies are therefore more susceptible to artefactual noise than other fMRI scenarios, the magnitude and nature of which are likely to depend on the exact experimental setup within each Lab, for each experiment, across participants. This highlights the importance of having a replicable set-up with properly placed and shielded electrode cables and stimulation equipment within the scanning area. For example, one study demonstrated evidence of BOLD signal within brains of two cadavers during a concurrent tDCS and fMRI protocol (Antal et al., 2014). Whilst a previous study from our Lab demonstrated visual evidence of change in echo-planar imaging (EPI) field maps that was limited to the scalp/surface near to the electrode site (Holland et al, 2011). These contrasting cases demonstrate the need for careful consideration of concurrent tDCS-fMRI data, and acquisition of appropriate field map data to allay concerns over false positive functional results from perturbation of the magnetic field.

To address this, prior to scanning human participants, we recommend a control tDCS concurrent with fMRI study for evaluation of the set-up in each Lab. The purpose of the control study is twofold: (i) to ensure the safety of concurrent tDCS and fMRI and (ii) to quantify any noise effects in the images induced by tDCS delivered simultaneously with the task stimuli. For example, in a previous experiment we delivered $2 \mathrm{~mA}$ anodal tDCS stimulation for 20 minutes concurrently with the identical stimulus delivery set-up as used in the main study to an inert object (a watermelon) (Holland et al., 2011). Results indicated that (i) during stimulation no significant changes in surface temperature were detected over time; and (ii) in 
distortion correction field maps only minimal perturbation of signal was observed at the electrode site (see Figure 4).

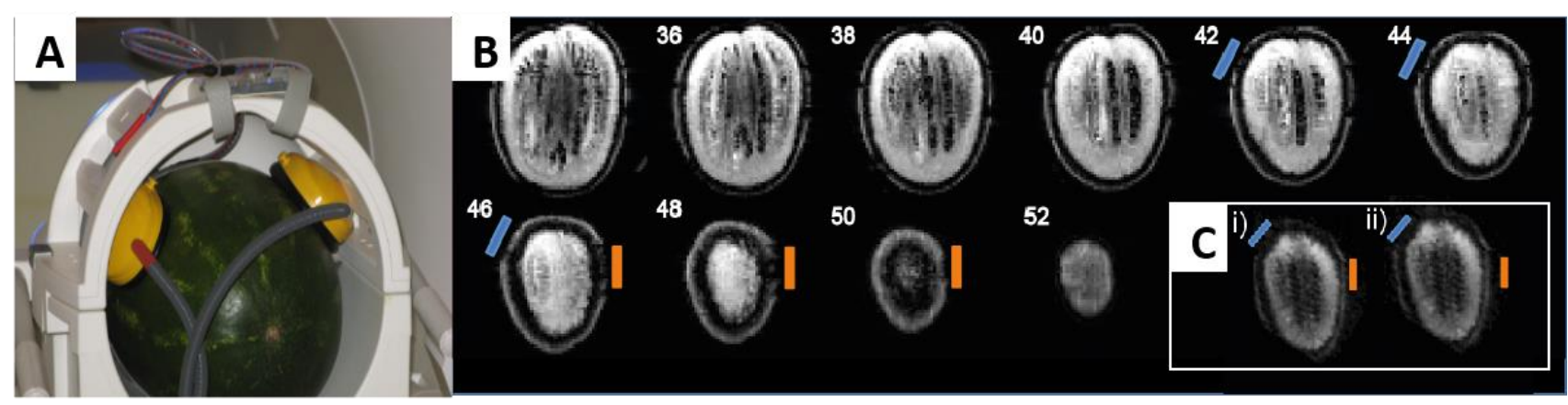

Figure 4 - Control concurrent tDCS and fMRI study. (A) A watermelon of similar size to a human adult head was chosen as a continuous $2 \mathrm{~mA}$ anodal DC could be passed through the surface. The headphones and tDCS electrodes were positioned on the object in the same orientation, and with the same tDCS/ fMRI set-up as was used for the main human study (cf. Holland et al., 2011); (B) Multi-slice coronal view of the watermelon field distortion.

The blue bar indicates the location of the anode electrode, orange the cathode electrode where mild perturbation of the signal is evident in slices $42-50$ at the surface layer of the watermelon under each electrode; (C) EPI from slice 46 (watermelon data) for i) un-stimulated (sham) and ii) stimulated (A-tDCS) fMRI runs. This figure has been used with permission from Holland et al., 2011.

The signal distortion was restricted to the surface of the watermelon only. Processing of the acquired functional data found no effects of tDCS on sham or stimulated runs. Together, these results indicated no imaging artefacts induced by the tDCS/fMRI set-up that could account for the effects of A-tDCS reported in the subsequent human experimental study (cf. Holland et al., 2011 for full details of effects of tDCS on EPI data). A control study such as this, comparing fMRI data for a short duration of time under two tDCS conditions, e.g., anodal vs sham, can be adapted for any experimental paradigm and tDCS-fMRI set-up.

In the human validation study presented here, the variability introduced by the tDCS equipment appears to be far below the level of variability arising from participant repositioning, reaffirming the importance of careful positioning. Generally higher $t$-score of the mean values were observed for the Anodal-tDCS case (cf. Figures 1 and 3). However, given that we would not expect an increase in functional sensitivity when using tDCS, this is more likely to originate from variability in participant repositioning, and scanner adjustments, across the different experimental conditions. Indeed, in the Anodal-tDCS case, the participant was positioned furthest into (superiorly) the sensitive volume of the receiving coil, boosting sensitivity. The cathode ROI (FP2) had greatest field inhomogeneity regardless of imaging condition. A frequency offset was observed in this ROI for the Anodal-tDCS case, however, there was no broadening of the frequency distribution. This indicates that the introduction of the tDCS equipment did not increase the field inhomogeneity. In fact, the broadest distribution was observed in this ROI for the No-tDCS condition. 


\section{Conclusion}

In this paper, we deliver the Wellcome Centre for Human Neuroimaging standard operating protocol (SOP) for technically sound and safe application of tDCS concurrently with fMRI. Although the MR-compatible tDCS technique is seemingly simple and easy to apply, we discuss specific aspects that must be taken into consideration when integrating the approach into an MRI environment to obtain both a safe experimental set-up and reliable results. This SOP and the experimental data validating its efficacy is provided as a detailed framework to systematically report the main technical elements of tDCS-fMRI, which can be adopted and used as a baseline for prospective real-world applicability. It is hoped that this will enhance the quality of tDCS-fMRI application in future studies, help provide practical solutions to the technical challenges and complications of the method, and therefore improve the quality of scientific work in this field further. 


\section{References}

Abellaneda-Perez, K., Vaqué-Alcázar' L., Perellón-Alfonso., R., et al. P275 Multifocal tDCS modulates resting-state functional connectivity in the elderly depending on baseline connectivity and induced electric field. Clin Neurophsiol. 2020; 131(4): e170-e172. Full $\underline{\text { Text }}$

Antal, A., Alekseichuk, I., Bikson, M., et al. Low intensity transcranial electric stimulation: Safety, ethical, legal regulatory and application guidelines. Clin Neurophysiol. 2017; 128(9): 1774-1809. PubMed Abstract | Full Text

Antal., A., Bikson, M., Datta, A., et al. Imaging artifacts induced by electrical stimulation during conventional fMRI of the brain. J Neurosci. 2014. 33(28); 11425-31. PubMed Abstract $\mid$ Full Text

Bikson, M., Grossman, P., Thomas, C et al. Safety of Transcranial Direct Current Stimulation: Evidence Based Update 2016. Brain. Stimul. 2016; 9 (5), 641-661. PubMed $\underline{\text { Abstract }} \mid \underline{\text { Publisher Full Text }}$

Brunoni, A.R., Sampaio-Junior, A., Moffa, A.H. Non-invasive brain stimulation in psychiatric disorders: a primer. Braz J Psychiatry. 2019; 41(1): 70-81. PubMed Abstract | Publisher Full Text

Chib, V.S., Yun, K., Shimojo, S. Noninvasive remote activation of the ventral midbrain by transcranial direct current stimulation of prefrontal cortex. Transl Psychiatry. 2013; 3(6): e268. PubMed Abstract | Publisher Full Text

Corbin N, Todd N, Friston KJ, Callaghan MF. Accurate modeling of temporal correlations in rapidly sampled fMRI time series. Hum Brain Mapp. 2018;39(10):3884-3897. PubMed Abstract $\mid \underline{\text { Publisher Full Text }}$

DaSilva, A., Volz, M., Bikson, M., Frehni, F. Electrode Positioning and Montage in Transcranial Direct Current Stimulation. J Vis Exp. 2011; 51: 2744. PubMed Abstract | Publisher Full Text

Datta., A., Bikson, M., and Fregni, F. Transcranial direct current stimulation in patients with skull defects and skull plates: high-resolution computational FEM study of factors altering cortical current flow. Neuroimage. 2010; 52(4): 1268-78. PubMed Abstract | Free Full Text

Esmaeilpour, Z., Shereen, A.D., Ghobadi-Azbari, P., et al. Methodology for tDCS integration with fMRI. Hum Brain Mapp. 2020; 41(7); 1950-1967. PubMed Abstract | Publisher Full Text 
Fertonani, A., Ferrai, C., Miniussi, C. What do you feel if I apply transcranial electric stimulation? Safety, sensations and secondary induced effects. Clin Neurophysiol. 205; 126(11): 2181-8. PubMed Abstract | Full Text

Ghobadi-Azbari,P., Jamil, A., Yavari, F., et al. fMRI and Transcranial Electrical Stimulation (tES): A systematic review of parameter space and outcomes. medRxiv: 2020. Publisher Full Text

Holland, R., Leff, A.P., Josephs, O, J., et al. Speech facilitation by left inferior frontal cortex stimulation. Curr Biol. 2011; 21(16): 1403-7. PubMed Abstract | Publisher Full Text

Meinzer, M., Lindenberg, R., Darkow, R., et al. Transcranial direct current stimulation and simultaneous functional magnetic resonance imaging. J. Visual. Exp: 2014; e51730. $\underline{\text { PubMed Abstract | Publisher Full Text }}$

Mondino, M., Ghumman, S., Gane, C., et al. Effects of Transcranial Stimulation With Direct and Alternating Current on Resting-State Functional Connectivity: An Exploratory Study Simultaneously Combining Stimulation and Multiband Functional Magnetic Resonance Imaging. Front. Hum. Neurosci. 2020; 5(13); 474. PubMed Abstract | $\underline{\text { Publisher Full Text }}$

Nitsche, M. A, Cohen, L.,Wassermann, et al. Transcranial direct current stimulation: State of the art 2008. Brain Stimul: 2008; 1, 206-223. PubMed Abstract | Publisher Full $\underline{\text { Text }}$

Nitsche, M.A., Bikson, M., Bestmann, S. On the Use of Meta-analysis in Neuromodulatory Non-invasive Brain Stimulation. Brain Stimul. 2015; 8(3); 666-7. PubMed Abstract | Publisher Full Text

Ondobaka, S., Nardo, D., Pappa, K., et al. P210 Using neuronal activity and fluid intelligence to understand individual differences in anodal tDCS modulation of spoken language. Clin Neurophysiol. 2020; 4: e135-136. Full Text

Paus, T. Inferring causality in brain images: a perturbation approach. Philos Trans $R$ Soc Lond B Biol Sci. 2005; 360(1457): 1109-14. PubMed Abstract | Publisher Full Text

Poreisz, C., Boros, K., Antal, A., and Paulus, W. Safety aspects of transcranial direct current stimulation concerning healthy subjects and patients. Brain Res Bull. 2007; 72(46): 208-14. PubMed Abstract | Full Text

Schulz, R., Gerloff, C., Hummel, F. Non-invasive brain stimulation in neurological diseases. Neuropharmacology. 2013; 64: 579-587. PubMed Abstract | Free Full Text 
Stagg, C.J., Lin, R.L., Mezue., M., et al. Widespread Modulation of Cerebral Perfusion Induced during and after Transcranial Direct Current Stimulation Applied to the Left Dorsolateral Prefrontal Cortex. J Neurosci. 2013; 33(28); 11425-11431. PubMed Abstract | Publisher Full Text

Thair, H., Holloway, A.L., Newport, R., Smith, A.D. Transcranial Direct Current Stimulation (tDCS): A Beginner's Guide for Design and Implementation. Front. Neurosci: 2017; 11(641). PubMed Abstract | Publisher Full Text

Woods, A.J., Antal, A. Bikson, M., et al. A technical guide to tDCS and related noninvasive brain stimulation tools. Clin Neurophysiol: 2016; 127(2), 1031-1948. PubMed Abstract $\mid$ Full Text 


\title{
Extended Data 1 - Standard Operating Procedure
}

\author{
Procedure pre-requisites
}

\section{Contraindications and special considerations}

When tDCS is integrated with MR, participant safety standards for both MR and tDCS should be adhered to as follows:

1) Participants should be screened using the safety checklist for MRI contraindications (e.g., any metallic implants including intracranial electrodes, cardiac pacemaker, shrapnel, surgical clips etc. susceptible to electrical current or magnetic fields) prior to recruitment.

2) Participants to be screened for any additional tDCS-specific contraindications - these contraindications may be study-specific. This includes presence of severe or frequent headache, any scalp or chronic skin condition: e.g., psoriasis or eczema where tDCS could aggravate the condition, or adverse reactions to a previous tDCS treatment. History of seizure and brain injury are usually not strict contraindications in themselves - for example, history of stroke is an inclusion criteria in our clinical studies, in patients who have been 1 year seizure free. Thair and colleagues (c.f., Thair et al., 2017 ) in their tDCS beginner's implementation guide have summarized general tDCS participant exclusion criteria and provided helpful Screening Questionnaires (c.f., Thair et al., 2017). Dedicated safety assessment should specifically be made for any indwelling medical devices and metallic implants (even if MRI compatible) that may change tDCS current flow (see Datta et al., 2010 for details).

\section{Areas and environments}

\section{Control Room}

This is the area that contains the operating PC for the scanner and the peripheral testing equipment. This falls within the outer-controlled area of the MRI environment. Consequently, personnel working in this environment must be trained in accordance with local policy. Participants entering this area must have been screened in accordance with local policy.

\section{Scan Room}

This is the room that contains the MRI system. This falls within the inner-controlled area of the MRI environment. Personnel working in this environment must be trained in accordance with local policy. Participants entering this area must have been screened in accordance with local policy.

\section{Testing Room}

This is an area external to the MRI environment that offers suitable facilities to prepare the participant for an MRI scan with tDCS. Ideally, this will offer privacy, must be large enough for three persons and provide adequate seating to allow for tDCS set-up, application and impedance testing. 


\section{MRI-compatible non-invasive brain stimulation system:}

We use the NeuroConn DC-stimulator (Extended Data Figure 1) https://www.neurocaregroup.com/dc_stimulator_mr.html

- MRI-compatible tDCS equipment:
a. Electrodes
b. Electrode cable
c. Inner- Box
d. Box cable (Ethernet cable)

- Non MRI-Compatible tDCS equipment:

e. Outer-Box

f. Stimulator cable

g. DC-Stimulator (NeuroConn)

Items e-g should be placed in a radiofrequency (RF) shielded box (Faraday cage) in the Control Room. The cables are connected via a waveguide between the Control Room and the Scan Room.

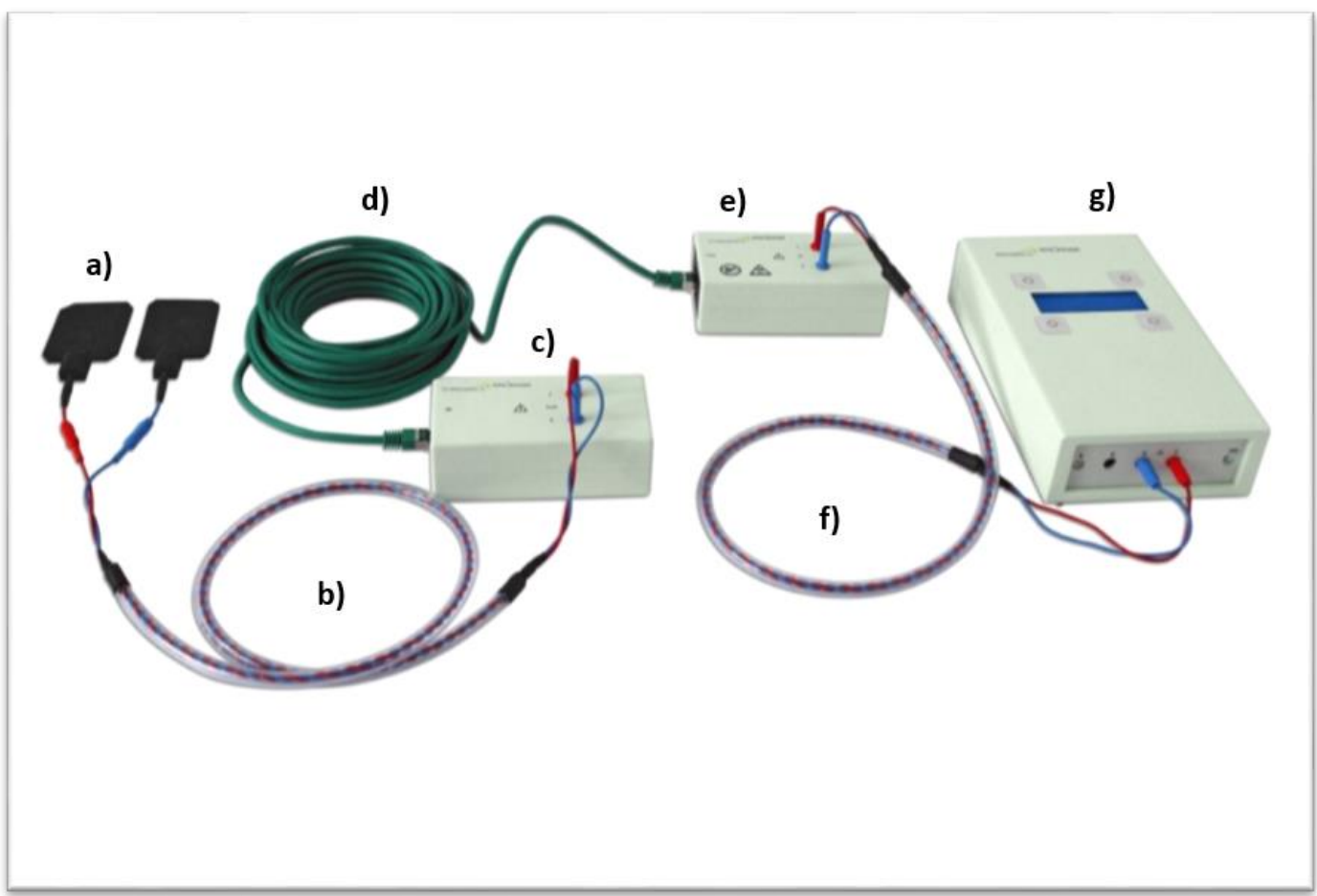

Extended Data Figure 1 - tDCS equipment. MRI-compatible equipment: (a) rubber electrodes, (b) twisted electrode cable with current limiting resistors 5,4 kOhm, (c) Inner-Box, (d) box cable. Non-MR-compatible equipment: (e) Outer-Box with attenuation circuit to eliminate RF interferences from outside the scanner 10-300 MHz, (f) twisted stimulator cable, (g) DC-Stimulator PLUS. Picture copyright: neuroCare Group GmbH 2020.

\section{IDCS contact medium and electrode placement}

To provide uniform current distribution throughout the prolonged fMRI experiment we use:

(i) EEG conductive paste (e.g., Ten20) as the MR electrode contact medium rather than saline soaked sponges or low viscosity electrode gel. This avoids premature drying which would result in an increase in resistance and risk to the participant of pain and skin burning. Using 
electrical conductance paste avoids these risks and the adhesive quality of the paste assists in affixing the electrode to the targeted location/scalp.

(ii) 3M Coban elastic wrap bandage to also comfortably and fully secure the electrode placement. Both the paste and bandage together improve stability of the scalp contact with the tDCS MR electrode even during our prolonged (i.e., $40 \mathrm{~min}$ ) overt speech fMRI paradigms that have higher associated head movements.

\section{RF Shielded Box}

In the Control Room, we installed a bespoke RF Shielded Box - this box is made from aluminium by the UCL Department of Chemistry, it was lined with beryllium copper shielding from RS Components (www.uk.rs-online.com/web/p/shielding-strips) (Extended Data Figure 2.A). The box is secured on a wooden shelf next to the waveguides. The box houses the DCStimulator (Extended Data Figure. 2. B), a Fibre-Optic Trigger Box to start the stimulator, and the leads connecting the stimulator to an RF filter box (Outer-Box, Extended Data Figure 1.e) which is placed in the waveguide. The addition of the RF shielded box was required in our laboratory to minimise any RF interference between the Scan Room and the external environment, with a 3 Tesla MR scanner in the adjacent room. What is important here is that this equipment doesn't act as a conduit causing RF to leak from the room and that the equipment doesn't allow too much, if any, noise into the room to degrade the fMRI.

The lid is kept on during the scanning and a small window in the lid allows the stimulator display screen to be monitored to ensure it is functioning correctly. The DC-Stimulator is removed at the end of each session, while the RF Shielded Box containing only the Fibre-Optic Box, the leads and the outer-box in the waveguide are left in place.

$\mathbf{A}$

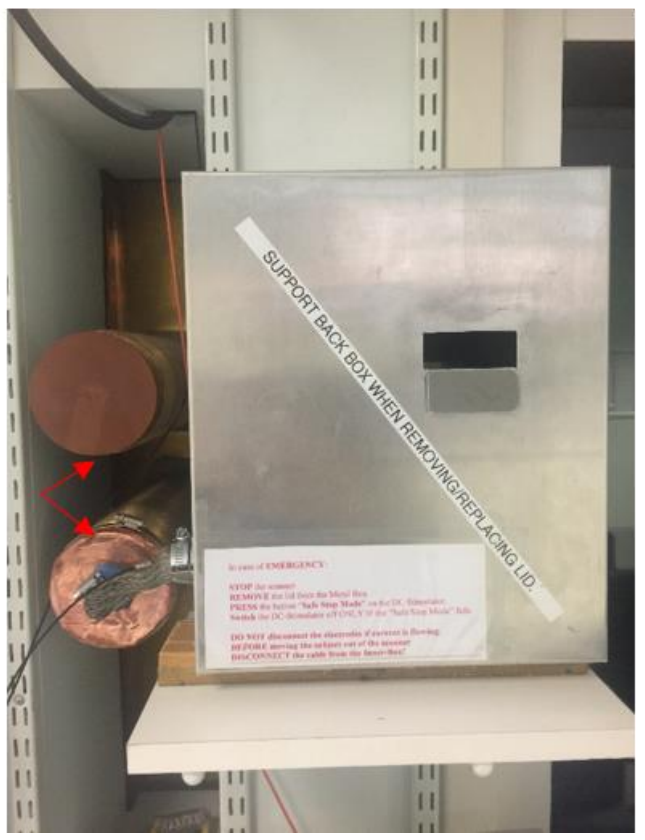

B

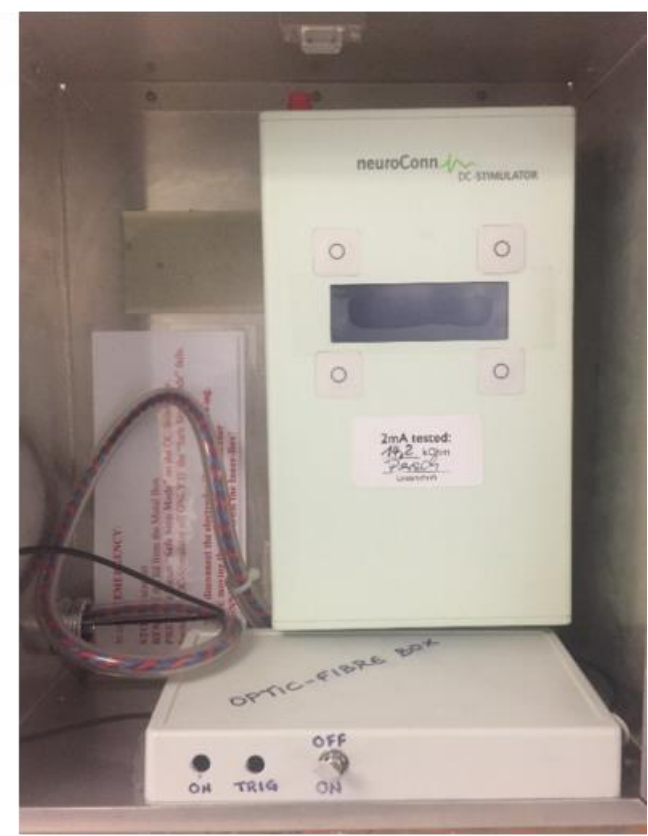

Extended Data Figure 2 - RF Shielded Box. (A) RF Shielded Box with lid on - the red arrows indicate the waveguides. (B) Placement of the DC Stimulator and Fibre Optic Box inside the RF Shielded Box. 


\section{Fibre-Optic Trigger Box}

The Fibre-Optic Trigger Box was built in-house at the WCHN; it is powered by a 9V battery and seated with Velcro within the RF Shielded Box (Extended Data Figure 2). It is used to electrically isolate the NeuroConn DC-Stimulator from the Stimulus PC, preventing the transmission of RF noise into the RF Shielded Box. The Stimulus PC generates a fibre-optic signal and the Fibre-Optic Trigger Box converts this signal into an electrical trigger, enabling operation of the NeuroConn DC-Stimulator via the Stimulus PC.

Additional items need for the Trigger Box:

(i) Trigger leads

(ii) Fibre optic cable (https://uk.rs-online.com/web/)

\section{Foam-base}

The tailor-made Foam-base assembled in-house at WCHN (Extended Data Figure 3) comprises:

- An MR bore-shaped foam block (black) purchased from Drayton Foam (http://www.draytonfoam.co.uk), with blue plastic components fixed on top of it and a plastic underlay (not shown) so it sits neatly in MRI bore.

In operation, the Foam-base secures the Inner-Box is positioned both centrally and consistently within the bore of the MRI system. This careful and fixed positioning prevents cable loops forming, maximises the distance between the transmit coil and the tDCS leads and ensures equipment placement is consistently replicated across scanning sessions.

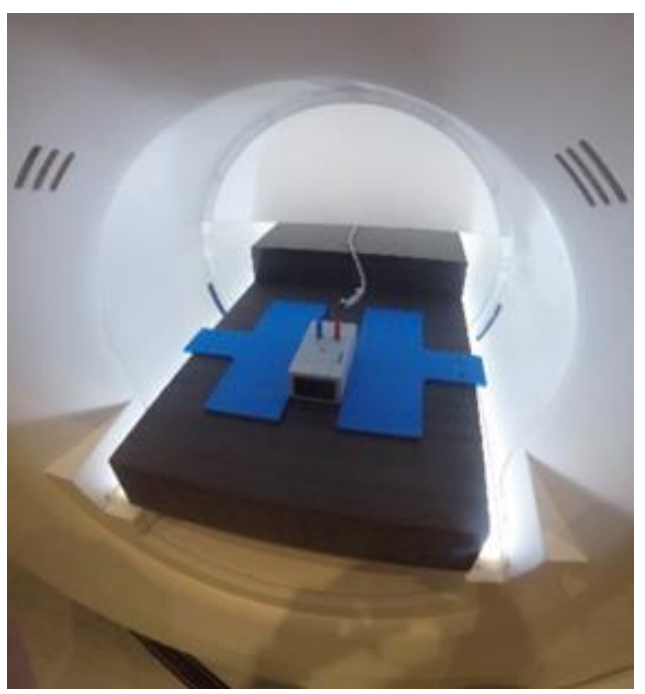

Extended Data Figure 3 - Foam-base for tDCS. Shown in the scanner bore with the InnerBox and tDCS electrode cabling in place.

6. MRI scanner

- Siemens Prisma 3 Tesla with 20 channel head coil 
The present protocol was optimised for a Siemens Prisma scanner. However, the NeuroConn DC-Stimulator is compatible with several models, and this fMRI-tDCS protocol can be recreated in any scanner through appropriate modification of the outlined procedures.

\section{Sandbags}

The tDCS box cable is run from the rear bore entry to the waveguide, hugging the perimeter of the room whenever possible. Siemens positioning sandbags are used to secure the box cable to the floor of the Scan Room to minimise the risk of creating a trip hazard. The sandbags are labelled 'Floor' and must not subsequently be used within the scanner bore.

\section{Stimulus $P C$}

For the experimental paradigm a computer that:

- Runs the software controlling the cognitive paradigm and therefore the NeuroConn DC-Stimulator (in these experiments this was MATLAB 2014a (The MathWorks, Inc., Natick, Massachusetts, United States) and COGENT 2000 developed by the Cogent 2000 team at the FIL and the ICN and Cogent Graphics developed by John Romaya at the LON at the Wellcome Department of Imaging Neuroscience.

- Receives MRI sequence triggers via a serial port.

- Outputs a fibre optic signal via parallel port to the Fibre-Optic Trigger Box.

\section{Paradigm-specific equipment}

This experiment delivered auditory stimuli and recorded overt spoken responses in the scanner using:

- An MR-compatible set of headphones (MR Confon, Magdeburg, Germany; www.mrconfon.de)

- A dual-channel, noise-cancelling fibre optical microphone system (FOMRI III, http://www.optoacoustics.com).

These are not essential for an fMRI-tDCS set up, and could easily be adapted for other cognitive paradigms.

\section{Persons and Responsibilities}

\section{Person 1}

Researcher primarily responsible for the tDCS equipment; this includes the setting up of equipment within the Scan Room and monitoring the Outer-Box during scanning.

\section{Person 2}

Researcher primarily responsible for the participant. Person 2 will take the lead in explaining the procedure to the participant, placing the electrodes, guiding the patient into the Scan room to test the electrodes, and helping Person 3 set up for the scan. Person 2 will also be responsible for disconnecting the electrodes within the Scan Room at the end of the scanning session. 


\section{Person 3}

A radiographer or Qualified User (QU) who will be present throughout the scan. They will be responsible for completing the MRI safety screening, participant care and positioning. During the scan Person 3 will also be responsible for operating the MRI scanner in accordance with the local rules. Including recording details of the imaging data acquisition, Specific Absorption Rate (SAR) levels and performing quality assurance of the images, in particular assessing them for evidence of participant movement and artefact, e.g. increased noise levels.

\section{Preparation}

Prior to beginning the imaging session, the Control Room, Scan Room and the participant must be suitably prepared. What follows is a detailed guide of how to do each.

\section{Control Room Preparation}

\section{Check connection at Fibre-Optic trigger box (Person 1)}

1. Ensure only one fibre-optic cable is entering the RF shielded box and interfacing with the Fibre-Optic Trigger Box.

2. This ensures RF integrity and electrical isolation.

\section{Check the 9V battery (Person 1)}

1. Ensure 9V battery is charged and fully operational (Extended Data Figure 4) by switching the small lever to the on position, both a red light (battery 'on' status) and a green light (trigger 'on' status) should come on.

2. If only the red light comes on, it means that the battery is charged but the trigger from the Stimulus PC is not correctly configured.

3. With our system, type 'outportb $(888,0)$ ' in the MATLAB command window (N.B: make sure the trigger is plugged in the parallel port of the Stimulus $P C$ ). This will ensure the DC-Stimulator is triggered to start when given the future MATLAB command during the experiment.

4. If no red light comes on, replace the battery.

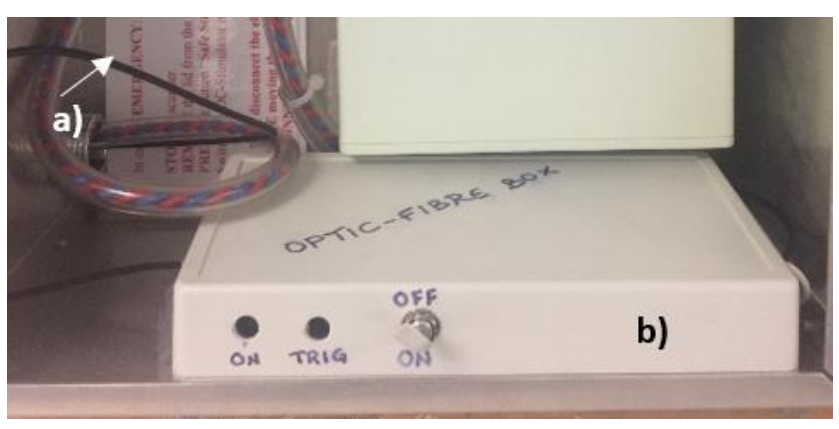

Extended Data Figure 4 - The Fibre-Optic Box and cables. (a) Fibre-Optic cable (black); (b) Fibre-Optic Trigger Box containing the $9 \mathrm{~V}$ battery. 
Secure the DC-Stimulator (Person 1)

1. Velcro strips are used to secure the DC-Stimulators' position inside the RF Shielded box (Extended Data Figure 2.b).

2. Connect the four jacks using their colour coded sockets as marked; red-anode (socket 1) and blue-cathode (socket 2) for electrodes, black (socket 3) and white (socket 4) for trigger (Extended Data Figure 5).

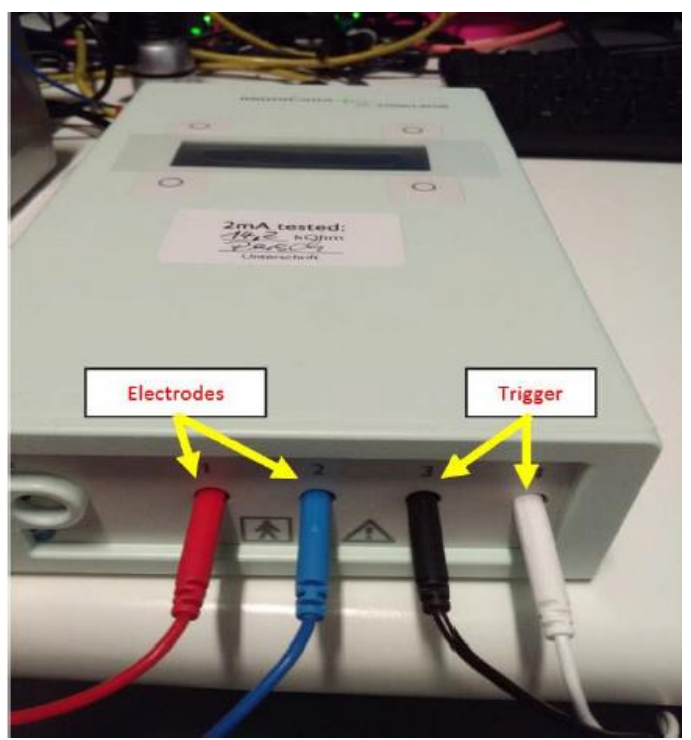

Extended Data Figure 5 - MR compatible DC-Stimulator connections (red-anode and blue-cathode for electrodes, black and white for trigger).

\section{Check ancillary equipment (Person 2)}

Other equipment that required setup for these particular experiments were:

- $\quad$ FOMRI Confon headphones and microphone

- $\quad$ Stimulus PC for the task

- $\quad$ Physiological data recording

\section{Scan Room Preparation}

\section{Position Foam-base for tDCS unit in the scanner bore (Person 1)}

1. Position and align the tailor-made Foam-base for tDCS in the rear section of the bore of the MRI scanner, aligning the posterior edges of the base and scanner (Extended Data Figure 6).

2. Place the Inner-Box onto the Foam-base between the blue support pads as shown in Extended Data Figure 3. This will ensure positioning is consistent throughout the experiments and across participants.

See Extended Data Figure 7 for a detailed schematic of the room layout and equipment positioning.

\section{Connect the box cable (Person 1)}

1. First, connect the box cable to the Inner-Box. 
2. The Outer-Box is kept inside the waveguide and positioned against the Control Room end of the waveguide. The Outer-Box contains ferrite components and therefore MUST NEVER enter the Scan Room.

3. To ensure the Outer-Box cannot be pulled into the Scan Room, as the cable is laid on the floor during room preparation, the Outer-Box is connected to the box cable LAST.

\section{Positioning the box cables (Person 1)}

1. The box cables should follow the path depicted in the room schematic (Extended Data Figure 7). These should run parallel to the bore, without loops and away from the participant to prevent the risk of eddy current induction and potential RF burns.

2. This cable runs from the Inner-Box inside the rear of the scanner bore to the back wall. It then follows the perimeter of the Scan Room (in this case the rear and left walls) to reach the Outer-Box in the waveguide.

3. It is positioned close to the wall edges, running as straight as possible without creating any loops. To achieve this, multiple sandbags and a cable protector are used as shown.

4. Once the participant is in position, they will be connected to the tDCS equipment. It is therefore important that the participant/scanner bed not be moved without disconnecting these. Tailor-made warning signs stating "CHECK electrode cables are disconnected before moving the bed" are therefore placed over the scanner controls (Extended Data Figure 8) for the duration of the scanning session.

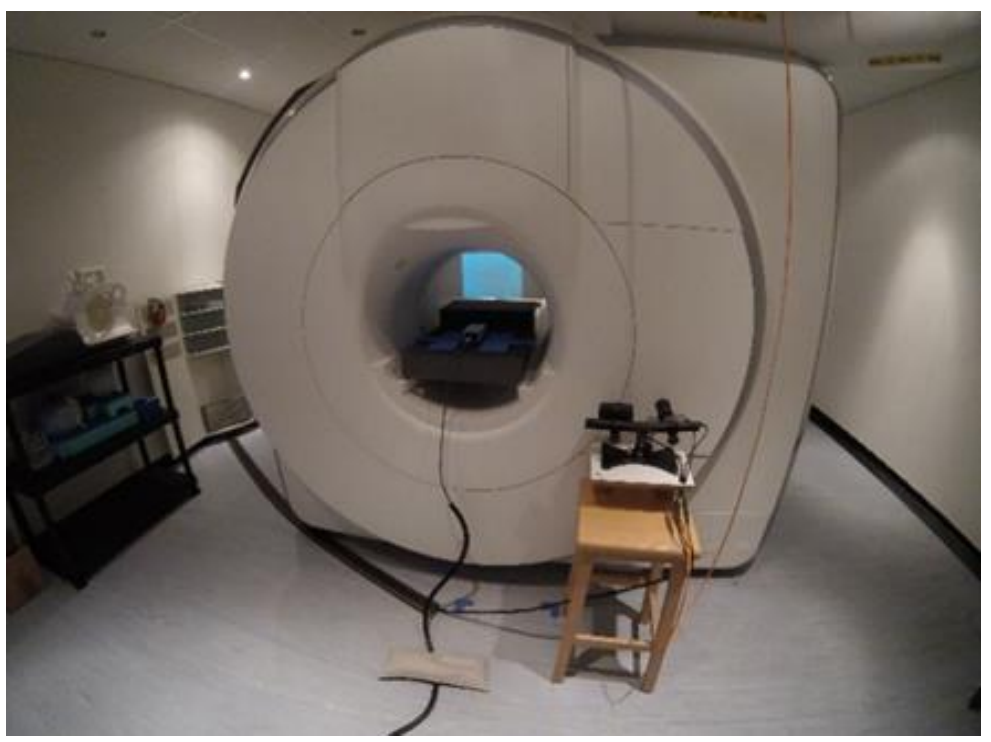

Extended Data Figure 6 - Route for Box Cables in scanner room. Photo taken from the back of the scanner: Foam-base in the scanner bore, with the Inner-Box on top. 


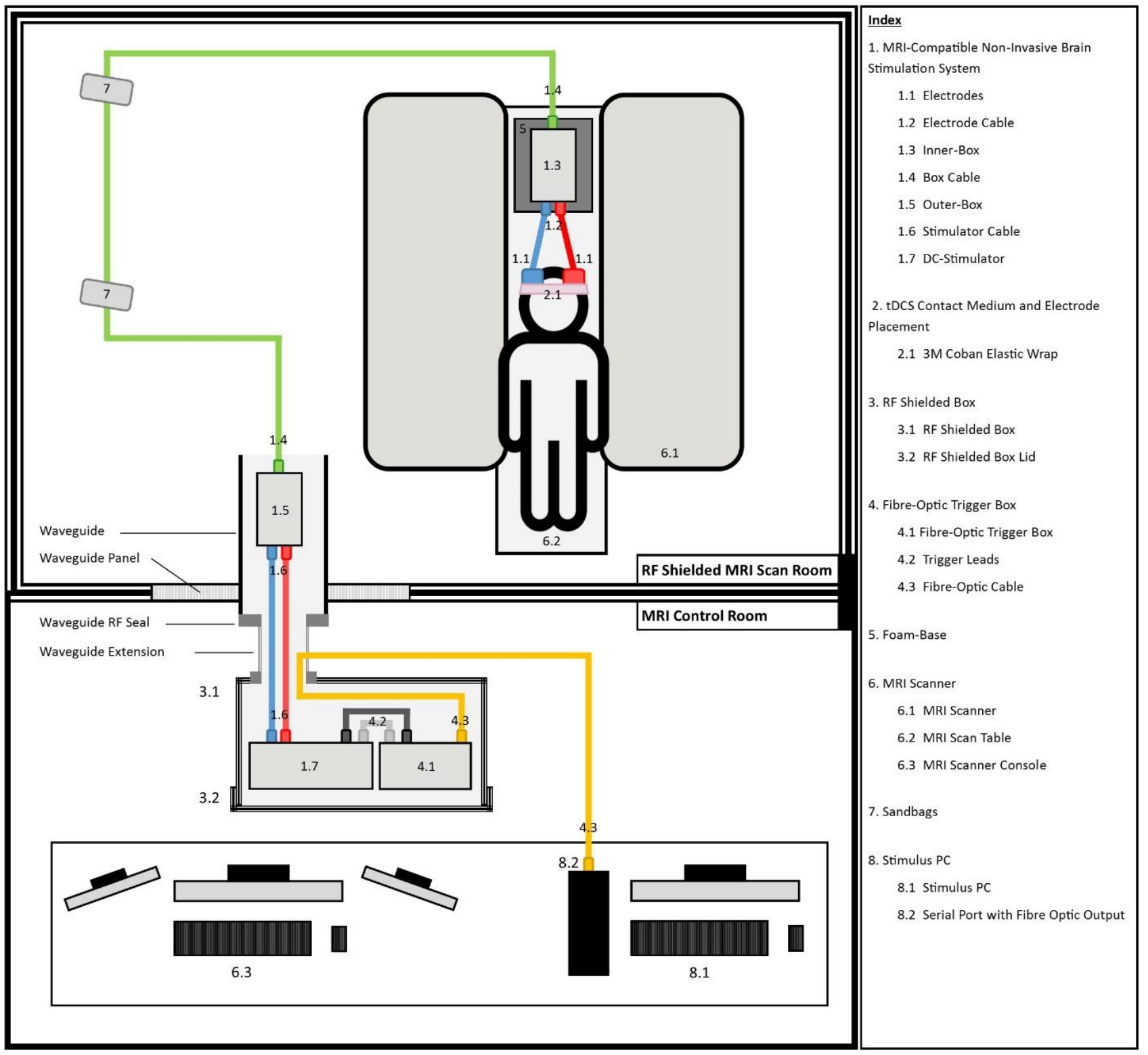

Extended Data Figure 7 - Schematic depicting full room layout. Image demonstrates the full set up of the tDCS and cable routes, with the participant positioned inside the scanner bore.
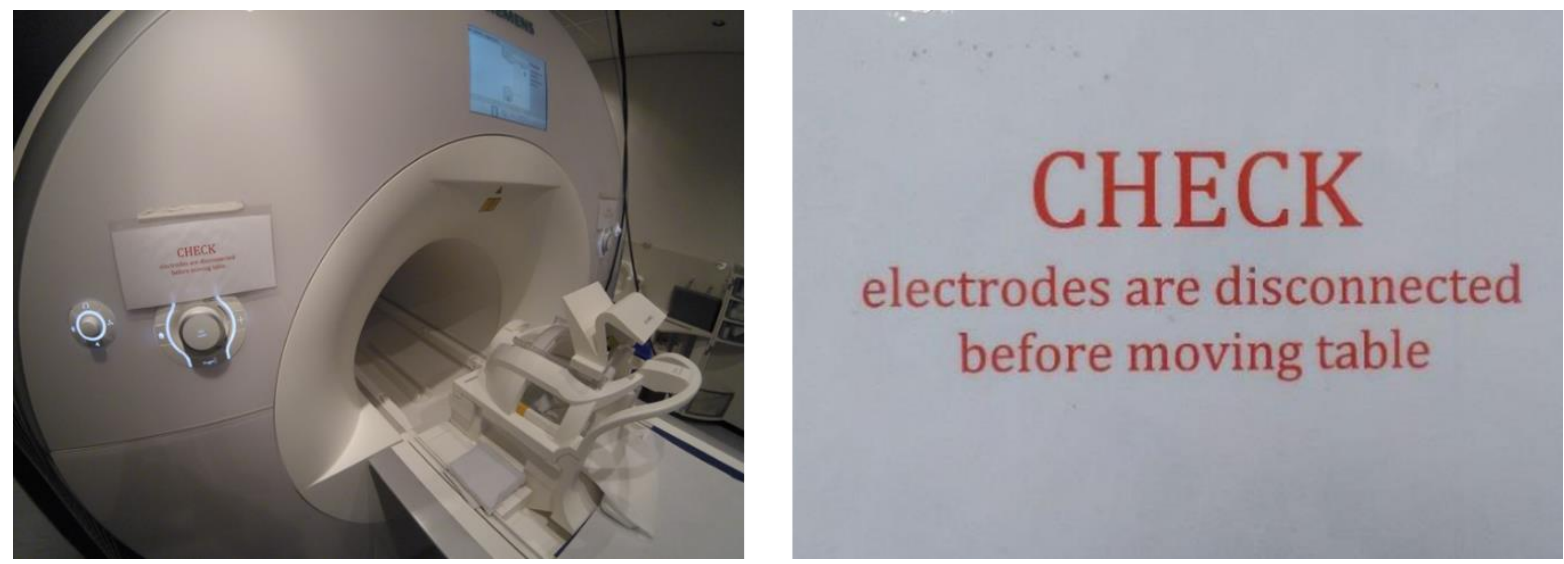
Extended Data Figure 8 - Warning Reminders. Photos taken from the front of the scanner. Warning signs to be put over the table controls on the scanner as a reminder not to move the bed/participant before disconnecting the electrode cables.

\section{Participant Preparation}

When tDCS is integrated with MR, participant safety standards for both MR and tDCS should be adhered to and participants pre-screened for both in advance of coming in for testing.

\section{Participant Safety Screening (Person 3)}

1. In the Testing Room, the radiographer will perform and cross-check the MR safety screening.

2. The participant should be ready to enter the MR environment (appropriate clothing, no jewellery, etc.).

3. Once the above is complete, tDCS safety screening is then cross-checked and completed.

4. When both safety checks are completed, then proceed with tDCS preparation.

tDCS Preparation (Person 2)

\section{MR electrode positioning}

In order to acquire good tDCS combined with MR data, careful preparation/placement of electrodes, stable fixation of the electrodes to the head, etc. remain critical considerations, as they are with tDCS alone (Woods et al., 2016). Scalp measurements are taken to ensure the correct placement of anodal and cathodal electrodes. The tDCS montage is chosen to accommodate the needs of the specific experimental procedure. In this case the stimulation site was the inferior frontal cortex (FC5 in a 10-20 EEG nomenclature) and was the site for the anodal electrode. The cathodal electrode was placed on the contralateral supra-orbital position (FP2; see Extended Data Figure 9) as in DaSilva (2011). However, the steps listed below can be applied to any tDCS montage regardless of the stimulation site and stimulation parameters.

1. Inspect the participants' skin to ensure there are no pre-existing lesions or allergies.

2. Clean the skin surface using suitable wipes.

3. Ensure any residue of face creams, hair products etc. is removed to ensure optimum conductivity.

4. Apply a layer of electrode-conductive paste (Ten20) on the MR biocarbon electrode surface. This should be a thick film $(\sim 3 \mathrm{~mm})$ of paste with thickness of paste prep kept consistent across sessions to provide sufficient distance between the electrodes and the scalp. This ensures that stimulation is delivered evenly across the electrode and also prevents direct contact between the skin and the electrodes, which is mandatory for functional safety and to prevent skin damage (Bikson et al., 2016).

5. Move participants' hair out of the way and apply pressure on the electrode when placing it on the skin/scalp. This ensures both MR-compatible electrodes are lying flat on the participants' skin surface for maximum comfort and achieving lower impedance.

6. The adhesive quality of the paste assists in affixing the electrode to the targeted location/scalp, but additional MR-compatible 3M Coban bandage wraps placed around 
the head, over the site of the electrodes, are also used for fully secure placement. This keeps the electrodes comfortably and securely in place (maximising skin contact and conductivity) throughout the experiment (Extended Data Figure 9).

A

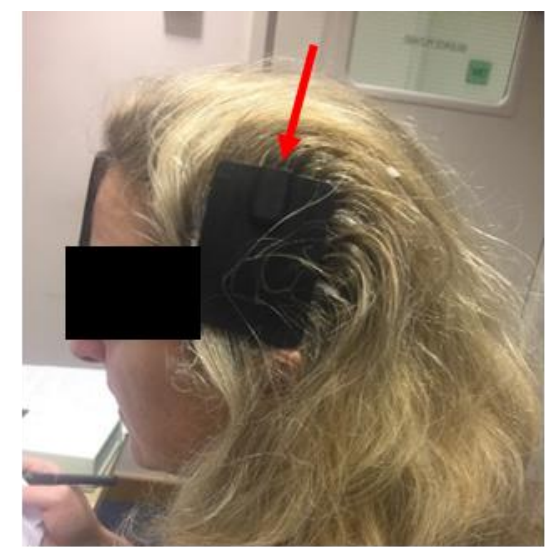

B

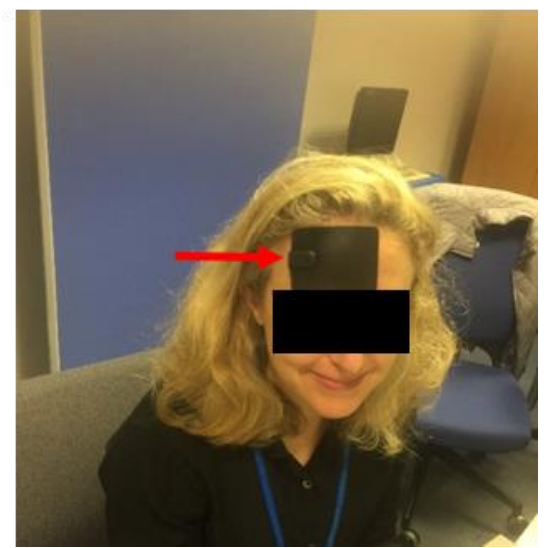

Extended Data Figure 9 - Electrode Placement. (A) Red arrow indicates placement of the anodal electrode on the participant's inferior frontal cortex (FC5) according to the 10-20 EEG nomenclature; (B) red arrow shows placement of the cathodal electrode on the contralateral frontopolar cortex (FP2).

\section{Impedance Check and 'tDCS taster' (Person 2, within the Testing Room)}

Impedance is checked to ensure there is good skin conductance and stability of electrode placement. The 'tDCS taster' allows the participant to experience the skin sensation when tDCS is delivered. This means they can more accurately: 1) rate the tDCS sensation strength, 2) ensure they are feeling comfortable with it, i.e. it is tolerable, and 3) agree they are willing to go ahead with the experimental procedure.

1. Attach the electrode cable leads to the electrodes.

2. Take care to ensure the electrode cable is straight, not creating any loops, not twisted nor braided. Place in adherence with the Manufacturers MR safety guidelines.

3. Attach the electrode cable to an external DC-Stimulator (i.e., a second non-MR DCStimulator different to the one in the RF Shielded box).

4. Turn the DC-Stimulator on with (for our experiment) the following 'tDCS-taster' parameters:
a. Duration: 60 seconds.
b. Fade In/Out: 15 seconds.
c. Current: $2 \mathrm{~mA}$.

5. Record the impedance displayed. The desired value within the Testing Room outside of the MR environment is $\leq 10 \mathrm{k} \Omega$. Wait for stimulation to finish.

6. Ask the participant to rate the sensation of the stimulation on a $0-10$ scale, 0 being nothing detected and 10 being unbearable.

a. $0-5=$ Proceed .

b. 6-10 = Ask participant to describe their feeling or discomfort. Ensure they are happy to proceed. 
Comfort ratings for this protocol show a favourable tolerability profile. Most often reported effects are tingling and itching sensations under the electrodes consistent with previous reported tolerable and minor tDCS effects (Poreisz et al., 2007, Fertonani et al., 2015). The sensation of phosphenes elicited by abrupt current on- or offset is avoided by the long ramping up of current intensity.

7. Repeat steps 1-6 if required for the participant to feel comfortable.

8. If the participant is not happy to proceed at any point, the experiment is stopped.

9. If the participant is happy to proceed, they can be taken into the MR Scan Room for the internal impedance check, having been MR safety screened (Person 3).

\section{Procedures within the MR Scan Room}

\section{Impedance Check}

As the MR-tDCS equipment has high-ohmic resistors in the stimulating circuit (in this case $10 \mathrm{kOhm}$ ) to prevent the induction of eddy currents within the stimulating leads, and the participant will have moved their head since leaving the Testing Room, the impedance is checked again in the Scan Room. This will ensure that the electrodes are still correctly in place and securely attached to the skin. This is done before the participant is positioned onto the scanner bed, so that any electrode placement issues can be rectified easily.

1. Attach the MR-compatible electrode cables to the electrodes on the participant's head - red anode, blue cathode (Person 2).

2. Slowly walk the participant to the rear of the scanner (Person 2).

3. Attach the MR-Compatible electrode cable to the DC-Stimulator via the Inner-Box, with the participant standing close to the back of the scanner bore (Person 2).

4. Person 2 should communicate to Person 1 when they are ready for the impedance check to begin.

5. Run the impedance test from the RF Shielded Box without delivering stimulation (Person 1).

6. Check the impedance is within an acceptable range (i.e., 10-20 k $\Omega$; cf. Woods et al., 2016) and record the number (Person 1).

7. If the impedance is not in range, check the electrodes to ensure that they have a secure fit to the scalp under, and only under, the electrodes with sufficient conductive paste evenly spread across the electrodes (Person 2).

8. Repeat until the desired range is achieved.

9. Switch off the DC-Stimulator in the RF-Shielded Box (Person 1).

10. Unplug cables from the Inner-Box but keep the electrode cables connected to the electrodes (Person 2).

11. Double check with the participant that they are happy to continue (Person 2). If they are not, terminate the experiment.

\section{Participant set-up for fMRI-tDCS}

1. Slowly guide the participant from the back of the scanner bore to the scanner bed.

2. Position the participant as per routine fMRI experimental protocols, but being careful that the electrodes do not move. It is vital to ensure that the electrodes are not in contact 
with the head coil, or headphones, to prevent electrode displacement and any unexpected interactions between the stimulator and the scanner. (Person 3).

3. Remind the participant that once the experiment starts, they may feel sensations from the tDCS stimulation (similar to experiences outside of the scanner during the tDCS 'taster' session, Person 2).

4. Remind the participant to squeeze the call bell at any time if they feel uncomfortable or want to stop, at which point they would be taken out of the scanner immediately (Person $3)$.

5. Once the anterior element of the head coil is in place, guide the electrode cables through openings in the head coil, exiting the right side of participant's headcoil in accordance with the manufacturer's DC stimulator MR application note: Single Channel fMRI-tES (https://www.neurocaregroup.com/dc_stimulator_mr.html).

6. The cable is lightly taped to the back of the head coil, taking care not to alter the positioning of the electrodes. This aims to ensure that the electrode cables do not get caught in the mechanisms of the bed as it moves to the scanner's isocentre (Person 2 and 3).

7. Move the participant to isocentre (Person 3).

8. Untape the electrode cables and connect them to the tDCS Inner-Box, which in our setup is on the Foam-base. Ensure there are no loops in the cable (Person 2).

9. The electrode cable is then taped to the Foam-base to be kept as straight as possible and centred with respect to the bore throughout the experimental procedure (Extended Data Figure 6).

\section{3. fMRI experiment procedure}

\section{Participant monitoring}

During the fMRI experiment, Person 2 constantly ensures that the participant is comfortable and at ease. To achieve the latter - for the purposes of this specific study - the participant is being monitored by two Scan Room cameras (front and back) and via a pulse oximeter. Furthermore, the participant is in constant contact with Person 2 through the optical FOMRI Microphone. However, the use of the microphone, cameras and the pulse oximeter are optional and not essential to conducting a tDCS-fMRI study.

\section{tDCS set-up prior to scanning (Person 1)}

1. Turn the Stimulator ON.

2. At this point the electrode cable and Inner-Box are fully connected; once more check the impedance is within the range specified (for our experiments we used a range of 10$20 \mathrm{k} \Omega$ ), and consistent with the impedance level noted during the internal impedance check (the check in the Scan Room prior to positioning for MRI).

3. Close the lid of the RF Shielded box within the Control Room.

\section{Delivering the stimulation}

1. Remind the participant of the sensation they may feel during the fMRI run (Person 2).

2. Provide the participant with instructions regarding the experimental tasks they are to carry out as part of the fMRI experiment (Person 2). 
3. Before each separate scanning run, check the participant is at ease and willing to continue.

4. When ready, indicate to Person 3 the scanning run can start (Person 2).

5. The stimulation is triggered by a scanner pulse at a given slice in the acquisition sequence.

6. Monitor the impedance throughout the whole experiment (Person 1).

7. Note what the impedance level is during the stimulation period (Person 1).

\section{MRI Quality Control (Person 3)}

1. Never shim or run sequences while the tDCS box is open.

2. Regularly check images for RF interference, with particular focus on background noise levels, which should be stable over time and of significantly lower signal intensity than voxels within the head.

\section{MRI Sequences}

The specific absorption rate (SAR), characterising power deposition and the associated risk of RF-induced heating, should be minimised during scanning. Standard 2D-Echo Planar Imaging (EPI) sequences, as used in the present studies, generally result in low SAR levels. However, the technique of multiband/simultaneous multi-slice, which is increasingly popular, will increase the SAR level. To enable correction of image distortions caused by inhomogeneity in the main magnetic field $\left(\mathrm{B}_{0}\right)$, field mapping data are often acquired. The standard, Siemensprovided (gre_field_mapping_1acq_rl), field mapping sequence was modified to reduce SAR in these studies by decreasing the excitation flip angle (from $90^{0}$ to $45^{\circ}$ ) as a further risk mitigation step.

\section{Performing a structural scan}

The participant should be taken out of the bore first, and disconnected from the tDCS equipment as the structural MRI sequences may have high SAR levels and the tDCS equipment is not necessary.

1. Disconnect the electrode cable carefully from in the Inner-Box - prior to moving the participant from the isocentre (Person 2).

2. Move the participant slowly out of the bore (Person 3).

3. Allow them to sit up and remove the electrode cable.

4. The MR electrodes and bandage may remain on during the structural scan.

5. Reposition the participant and perform the structural scan.

\section{Procedure end and equipment removal}

\section{Removing participant from the bore}

1. Switch the DC-Stimulator to OFF.

2. Disconnect the electrode cable from the Inner-Box BEFORE moving participant out of the scanner (Person 2).

3. Move the participant slowly from the scanner (Person 3). 
4. Remove the anterior element of the head coil carefully to avoid the electrodes getting caught.

5. Allow the participant to sit up, then remove the electrodes (Person 2).

6. Accompany the participant back to the Testing Room (Person 2).

Post-MRI (Person 2)

1. Ask the participant to rate the tDCS strength and report if they experienced any adverse sensations due to the tDCS stimulation. Importantly, from a research reproducibility perspective, with the equipment outlined in this protocol, sham conditions, where a few seconds of stimulation that does not change cortical excitability (Nitsche et al., 2008) but generates equivalent sensations for participants (itching, tingling etc.) can be achieved. This enables double blind experiments to be carried out.

2. Wash any paste from the electrodes and disinfect with appropriate wipes.

Post-MRI (Person 1)

1. Remove all equipment from the Scan Room and leave as found (reposition any equipment that was moved to facilitate the experiment).

2. Remove the electrode cable and Inner-Box from the bore, and store outside of the Scan Room in the manufacturers' storage case.

3. Remove the Foam-base and store securely in the Scan Room.

4. Remove the DC-Stimulator from the RF Shielded Box, and store together with the electrodes, electrode cable, and the Inner-Box outside of the Scan Room in the manufacturers' storage case.

5. Switch the Fibre-Optic Box off.

6. Close the RF Shielded box using lid (Extended Data Figure 2).

7. Leave the Outer-Box and stimulator cable inside the waveguide at all times - DO NOT REMOVE when leaving. The Outer-Box should ALWAYS be positioned at the Control Room end of the waveguide.

\section{Emergency Procedures}

Considering the complexity of conducting a concurrent fMRI-tDCS study, the present protocol provides guidelines on how to safely remove a participant in case they feel unwell or choose to terminate the experiment. If this happens while the stimulation is being delivered, there are specific steps that should be taken to enable the safe removal of the participant from the scanner bore. To ensure the efficient removal of a participant in case of emergency, each person present will perform a separate duty. A minimum of three people is required for safety reasons, i.e. a radiographer plus two experienced researchers.

1. Person 3 (radiographer) stops the scanner.

2. Person 2 informs the participant 'we are coming in'.

3. Person 1 removes the lid from the RF Shielded Box and if tDCS is still being delivered stops the stimulation by using the 'Safe Stop Mode' feature of the NeuroConn DC stimulator (Extended Data Figure 10), then switches the DC-Stimulator off. The DCStimulator should only be directly switched off if stimulation is not being delivered (i.e., already terminated) or in the (extremely unlikely) case that the 'Safe Stop Mode' fails.

4. Person 2 goes directly to the back of the scanner ready to disconnect the electrodes.

5. Person 3 goes into position to remove the participant from the scanner bore. 
6. Person 1 states loudly 'safe to disconnect' when the DC-Stimulator is off.

7. Person 2 then disconnects the electrode cable and states 'safe to move bed' to the radiographer. The electrodes are not to be disconnected if current is still flowing as this may cause a strong stimulus to be delivered (cf. pages 14-15 and 106-108 of NeuroConn DC- stimulator MR Manufacturer's Manual).

8. Person 3 then moves the bed and participant out.

9. In the unlikely event a trolley evacuation is deemed necessary, Person 1 and 2 will disconnect the box cable from the Outer-Box and remove the box cable from the evacuation doorway. Then normal trolley emergency procedure is followed.

A

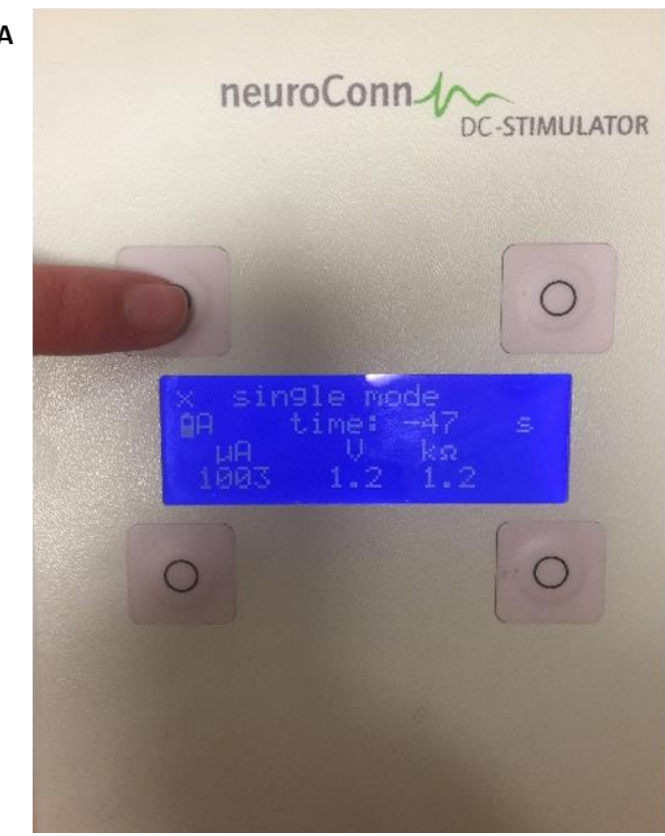

B

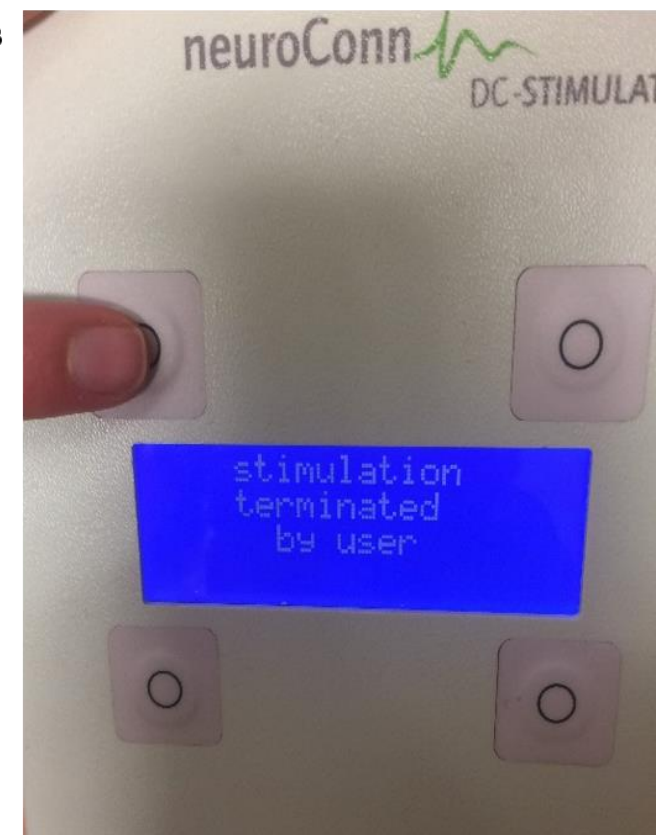

Extended Data Figure 10 - Safe Mode of tDCS. (A) 'Safe Stop Mode' (i.e., top-left) button to stop the DC-Stimulator in case of an emergency; (B) message of successfully terminated stimulation.

\section{THERE ARE NO FURTHER PROCEDURAL STEPS TO THIS DOCUMENT}




\section{Extended Data 2 - MatLab Script}

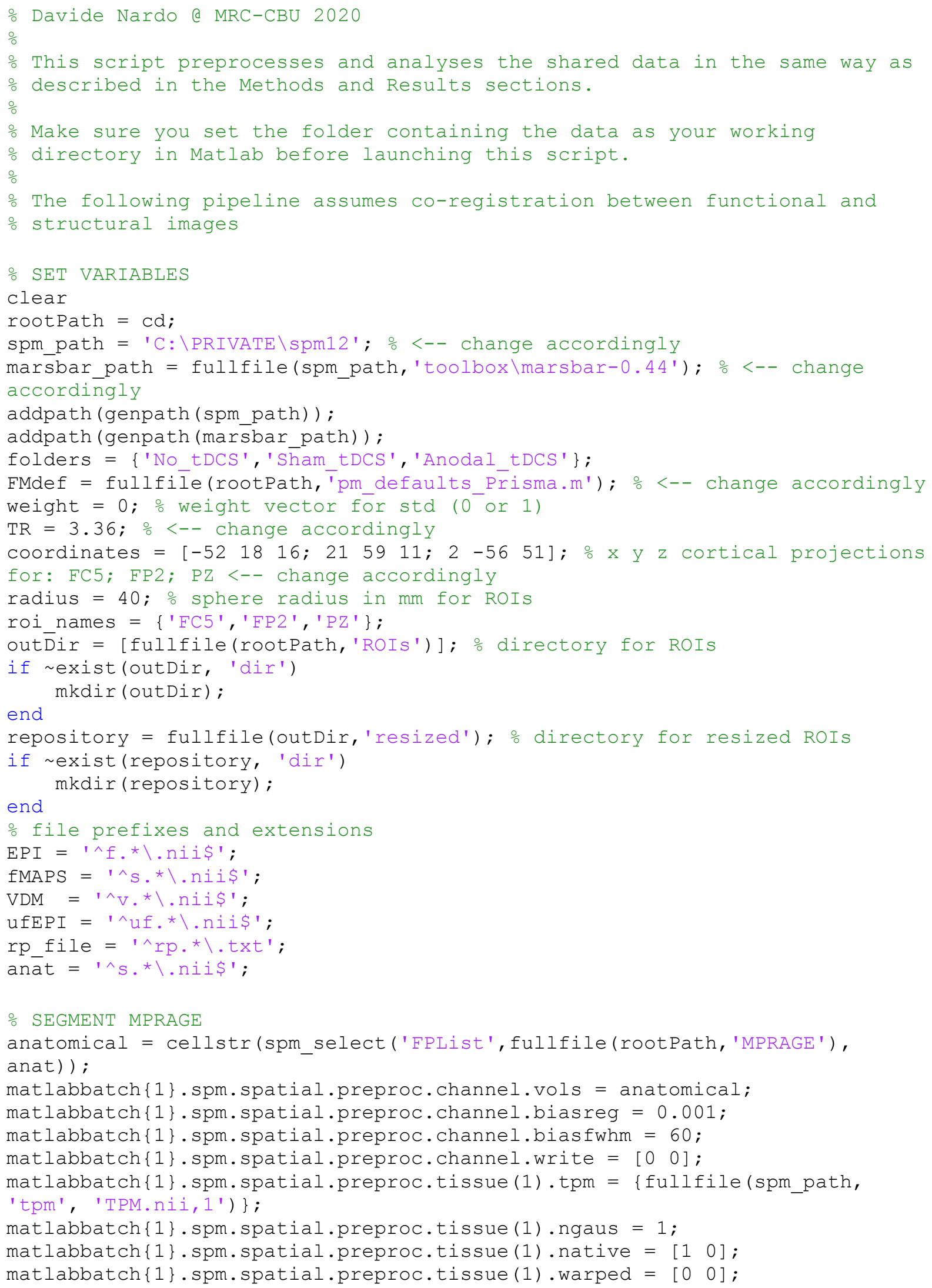

\author{
Pre-processing and data analysis
}


matlabbatch $\{1\}$.spm.spatial.preproc.tissue(2).tpm $=\{$ fullfile(spm_path, 'tpm', 'TPM.nii,2')\};

matlabbatch $\{1\}$.spm.spatial.preproc.tissue (2).ngaus $=1$;

matlabbatch $\{1\}$. spm.spatial.preproc.tissue (2). native $=\left[\begin{array}{ll}1 & 0\end{array}\right]$;

matlabbatch $\{1\}$.spm.spatial.preproc.tissue (2). warped $=\left[\begin{array}{ll}0 & 0\end{array}\right]$;

matlabbatch $\{1\}$.spm.spatial.preproc.tissue(3).tpm $=\{$ fullfile(spm_path, 'tpm', 'TPM.nii, 3')\};

matlabbatch $\{1\}$.spm.spatial.preproc.tissue(3).ngaus $=2$;

matlabbatch $\{1\}$.spm.spatial.preproc.tissue (3). native $=\left[\begin{array}{ll}1 & 0\end{array}\right]$;

matlabbatch $\{1\}$.spm.spatial.preproc.tissue (3) $\cdot$ warped $=\left[\begin{array}{ll}0 & 0\end{array}\right]$;

matlabbatch $\{1\}$.spm.spatial.preproc.tissue (4).tpm $=\{$ fullfile (spm_path,

'tpm', 'TPM.nii,4')\};

matlabbatch $\{1\}$. spm.spatial.preproc.tissue $(4)$.ngaus $=3$;

matlabbatch $\{1\}$.spm.spatial.preproc.tissue $(4)$.native $=\left[\begin{array}{ll}1 & 0\end{array}\right] ;$

matlabbatch $\{1\}$.spm.spatial.preproc.tissue $(4) \cdot \operatorname{warped}=\left[\begin{array}{ll}0 & 0\end{array}\right]$;

matlabbatch $\{1\}$.spm.spatial.preproc.tissue(5).tpm $=\{$ fullfile(spm_path, 'tpm', 'TPM.nii,5')\};

matlabbatch $\{1\}$.spm.spatial.preproc.tissue $(5) \cdot$ ngaus $=4$;

matlabbatch $\{1\}$.spm.spatial.preproc.tissue (5). native $=\left[\begin{array}{ll}1 & 0\end{array}\right]$;

matlabbatch $\{1\}$.spm.spatial.preproc.tissue (5). warped $=\left[\begin{array}{ll}0 & 0\end{array}\right]$;

matlabbatch $\{1\}$.spm.spatial.preproc.tissue (6).tpm $=\{$ fullfile (spm_path, 'tpm', 'TPM.nii,6')\};

matlabbatch $\{1\}$. spm. spatial.preproc.tissue $(6)$.ngaus $=2$;

matlabbatch $\{1\}$.spm.spatial.preproc.tissue $(6)$. native $=\left[\begin{array}{ll}0 & 0\end{array}\right] ;$

matlabbatch $\{1\}$.spm.spatial.preproc.tissue $(6) \cdot \operatorname{warped}=\left[\begin{array}{ll}0 & 0\end{array}\right] ;$

matlabbatch $\{1\}$.spm.spatial.preproc.warp.mrf $=1$;

matlabbatch $\{1\}$.spm.spatial.preproc.warp.cleanup $=1$;

matlabbatch $\{1\}$.spm.spatial.preproc.warp.reg $=\left[\begin{array}{llllll}0 & 0.001 & 0.5 & 0.05 & 0.2\end{array}\right]$;

matlabbatch $\{1\}$.spm.spatial.preproc.warp.affreg = 'mni';

matlabbatch $\{1\}$. spm.spatial.preproc.warp. fwhm $=0$;

matlabbatch $\{1\}$.spm.spatial.preproc.warp. samp $=3$;

matlabbatch $\{1\}$.spm.spatial.preproc.warp.write $=\left[\begin{array}{ll}0 & 0\end{array}\right]$;

matlabbatch $\{1\}$.spm.spatial.preproc.warp.vox $=\mathrm{NaN}$;

matlabbatch $\{1\}$.spm.spatial.preproc.warp.bb $=[\mathrm{NaN} N a N$ NaN; NaN NaN NaN];

spm_jobman ('run', matlabbatch);

clear matlabbatch

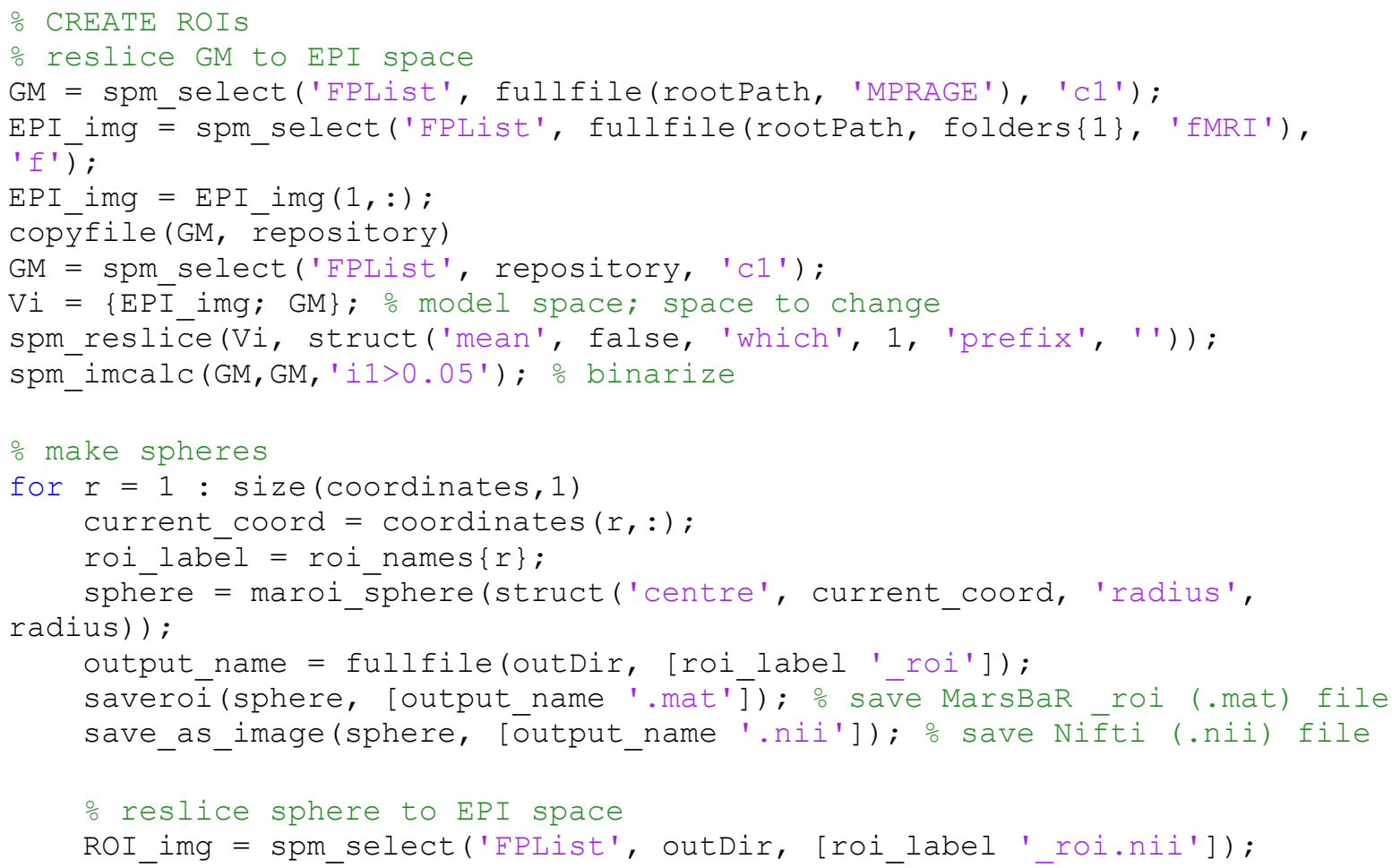


copyfile(ROI_img, repository)

ROI_img = spm_select('FPList', repository, [roi_label '_roi.nii']);

$\mathrm{Vi}=\{\mathrm{EPI}$ img; ROI_img $\} ;$ model space; space to change

spm_reslic̄e(Vi, struct('mean', false, 'which', 1, 'prefix', ''));

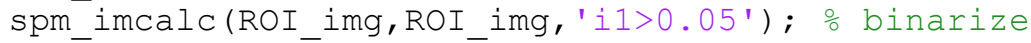

\% merge ROI \& GM

matlabbatch $\{1\}$.spm.util.imcalc.input $=\{\mathrm{GM} ; \mathrm{ROI}$ img $\}$;

matlabbatch $\{1\}$.spm.util.imcalc.output = ['GM_' roi_label];

matlabbatch $\{1\}$.spm.util.imcalc.outdir = \{repository $\}$;

matlabbatch $\{1\}$.spm. util.imcalc.expression = 'i1\&i2';

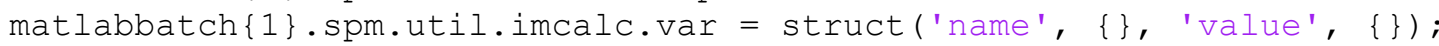

matlabbatch $\{1\}$.spm.util.imcalc.options.dmtx $=0$;

matlabbatch $\{1\}$.spm.util.imcalc.options.mask $=0$;

matlabbatch $\{1\}$.spm.util.imcalc.options.interp $=1$;

matlabbatch $\{1\}$.spm.util.imcalc.options.dtype $=4$;

spm_jobman ('run', matlabbatch);

clear matlabbatch

end

\% PREPROCESS DATA \& ANALYSE T-SCORE

for fold $=1$ : size(folders, 2$)$ : folders loop

current folder $=$ folders $\{$ fold $\}$;

\% fieldmapping

epiDir = cellstr(fullfile(current_folder,'fMRI'));

datafiles $\{1\}=\operatorname{cellstr}($ spm_select('FPList', epiDir, EPI));

fMaps $=$

cellstr(spm_select('FPList',fullfile(current_folder,'FieldMap'), fMAPS));

matlabbatch $\{1\}$.spm.tools.fieldmap.calculatevdm.subj.data.presubphasemag.pha se $=\{\operatorname{fMaps}\{3\}\} ;$

matlabbatch $\{1\}$.spm.tools.fieldmap.calculatevdm.subj.data.presubphasemag.mag nitude $=\{$ fMaps $\{1\}\}$;

matlabbatch $\{1\}$.spm.tools.fieldmap.calculatevdm.subj.defaults.defaultsfile = $\{$ FMdef $\}$;

matlabbatch $\{1\}$.spm.tools.fieldmap.calculatevdm.subj.session(1).epi = datafiles $\{1,1\}(1,:)$;

matlabbatch $\{1\}$.spm.tools.fieldmap. calculatevdm.subj.matchvdm = 1 ;

matlabbatch $\{1\}$.spm.tools.fieldmap.calculatevdm.subj.sessname $=$

'session' ;

matlabbatch $\{1\}$.spm.tools.fieldmap.calculatevdm.subj.writeunwarped = 1 ;

matlabbatch $\{1\}$.spm.tools.fieldmap.calculatevdm.subj.anat = ' ' ;

matlabbatch $\{1\}$.spm.tools.fieldmap.calculatevdm.subj.matchanat $=0$;

spm_jobman('run', matlabbatch);

clear matlabbatch epiDir datafiles fMaps

\% realign \& unwarp

epiDir = cellstr(fullfile(current folder,'fMRI'));

datafiles \{\}$=\operatorname{cellstr}($ spm_select('FPList', epiDir, EPI));

fMaps $=$

cellstr(spm_select('FPList',fullfile(current_folder,'FieldMap'), VDM)); matlabbātch $\{1\}$.spm.spatial.realignunwarp.data(1).scans =

datafiles $\{1,1\}$;

matlabbatch $\{1\} \cdot \operatorname{spm}$. spatial.realignunwarp.data $(1) \cdot \operatorname{pmscan}=\{\operatorname{fMaps}\{1\}\} ;$

matlabbatch $\{1\}$.spm.spatial.realignunwarp. eoptions.quality $=1$;

matlabbatch $\{1\}$.spm.spatial.realignunwarp.eoptions. $\operatorname{sep}=4$;

matlabbatch $\{1\}$.spm.spatial.realignunwarp.eoptions.fwhm $=5$; 
matlabbatch $\{1\}$.spm.spatial.realignunwarp.eoptions.rtm $=0$; matlabbatch $\{1\}$.spm.spatial.realignunwarp.eoptions.einterp $=2$; matlabbatch $\{1\}$.spm.spatial.realignunwarp.eoptions.ewrap $=\left[\begin{array}{lll}0 & 0 & 0\end{array}\right]$; matlabbatch $\{1\}$.spm. spatial.realignunwarp.eoptions.weight = ' '; matlabbatch $\{1\}$.spm.spatial.realignunwarp.uweoptions.basfen = [12 12]; matlabbatch $\{1\}$.spm. spatial.realignunwarp. uweoptions.regorder $=1$; matlabbatch $\{1\}$.spm.spatial.realignunwarp. uweoptions.lambda $=100000$; matlabbatch $\{1\}$.spm.spatial.realignunwarp.uweoptions.jm = 0 ; matlabbatch $\{1\}$.spm. spatial.realignunwarp.uweoptions.fot $=[45]$; matlabbatch $\{1\}$.spm.spatial.realignunwarp.uweoptions.sot = []; matlabbatch $\{1\}$.spm.spatial.realignunwarp.uweoptions.uwfwhm $=4$; matlabbatch $\{1\}$.spm.spatial.realignunwarp.uweoptions.rem = 1 ; matlabbatch $\{1\}$.spm.spatial.realignunwarp.uweoptions.noi $=5$; matlabbatch $\{1\}$.spm.spatial.realignunwarp.uweoptions.expround $=$

'Average' ;

matlabbatch $\{1\}$.spm.spatial.realignunwarp.uwroptions.uwwhich $=[2$ 1]; matlabbatch $\{1\}$.spm.spatial.realignunwarp. uwroptions.rinterp $=4$; matlabbatch $\{1\}$.spm.spatial.realignunwarp.uwroptions.wrap $=\left[\begin{array}{lll}0 & 0 & 0\end{array}\right]$; matlabbatch $\{1\}$.spm.spatial.realignunwarp.uwroptions.mask = 1; matlabbatch $\{1\}$.spm.spatial.realignunwarp.uwroptions.prefix = 'u'; spm_jobman('run', matlabbatch);

clear matlabbatch epiDir datafiles fMaps

\% create \& estimate GLM output_folder = fullfile(rootPath, current_folder, 'GLM'); epiDir = fullfile (rootPath, current folder,'fMRI'); datafiles = cellstr (spm_select('FPList', epiDir, ufEPI)); motion_params = spm_select('FPList', epiDir, rp_file);

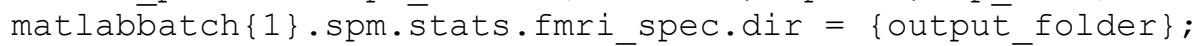
matlabbatch $\{1\}$.spm.stats.fmri_spec.timing.units $=$ 'scans'; matlabbatch $\{1\}$.spm.stats.fmri_spec.timing.RT = TR; matlabbatch $\{1\}$.spm.stats.fmri_spec.timing.fmri_t $=16$; matlabbatch $\{1\}$.spm.stats.fmri_spec.timing.fmri_to $=8$; matlabbatch $\{1\}$.spm.stats.fmri_spec.sess.scans $=$ datafiles; matlabbatch $\{1\}$.spm.stats.fmri_spec.sess.cond = struct('name', \{\} ,

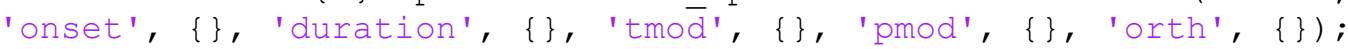
matlabbatch $\{1\}$.spm.stats.fmri_spec.sess.multi $=\left\{\prime^{\prime}\right\}$; matlabbatch $\{1\}$.spm.stats.fmri_spec.sess.regress $=\operatorname{struct}\left(\right.$ 'name' $^{\prime}\{\}$, 'val', \{\}); matlabbatch $\{1\}$.spm.stats.fmri spec.sess.multi reg $=$ \{motion params\}; matlabbatch $\{1\}$.spm.stats.fmri_spec.sess.hpf $=128$;

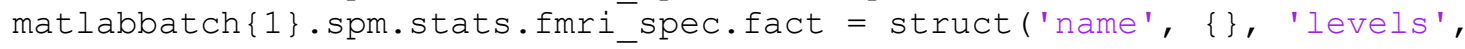
\{\}$)$;

matlabbatch $\{1\}$.spm.stats.fmri spec.bases.hrf.derivs $=\left[\begin{array}{ll}0 & 0\end{array}\right] ;$ matlabbatch $\{1\}$.spm.stats.fmri_spec.volt $=1$; matlabbatch $\{1\}$.spm.stats.fmri_spec.global = 'None'; matlabbatch $\{1\}$.spm.stats.fmri spec.mthresh $=1$; matlabbatch $\{1\}$.spm.stats.fmri_spec.cvi = 'AR(1)'; spm_jobman ('run', matlabbatch);

clear matlabbatch epidir datafiles motion params

\% estimate

estimate file = spm_select('FPlist', output_folder,'SPM.mat');

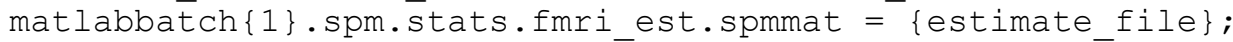
matlabbatch $\{1\}$.spm.stats.fmri_est.write_residuals $=\overline{0}$; matlabbatch $\{1\}$.spm.stats.fmri est.method. classical $=1$; spm_jobman ('run', matlabbatch); clear matlabbatch

\% compute contrast ( $\mathrm{T}$-score of the mean) contrast $=[\operatorname{zeros}(1,6) 1]$; 


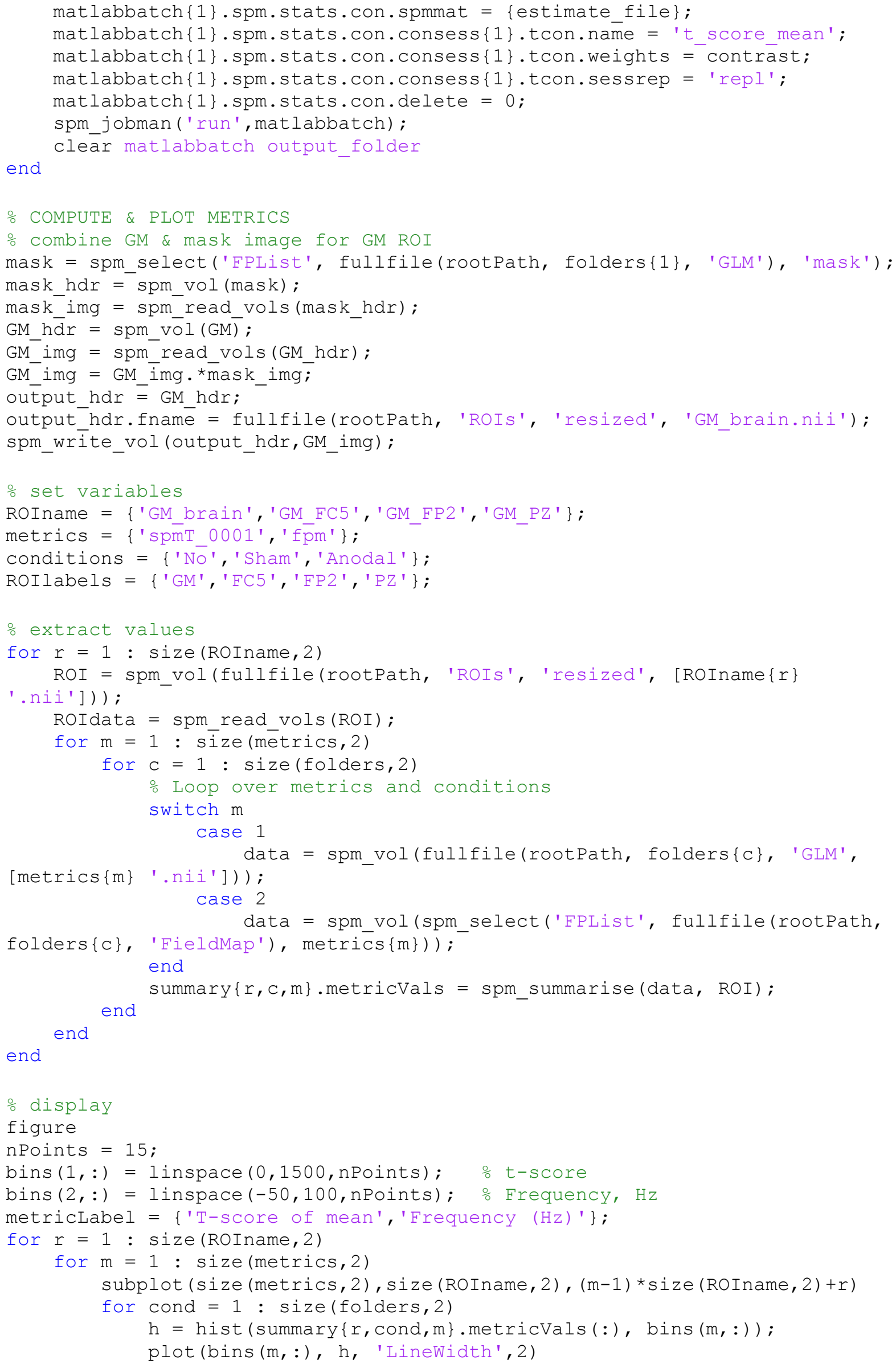




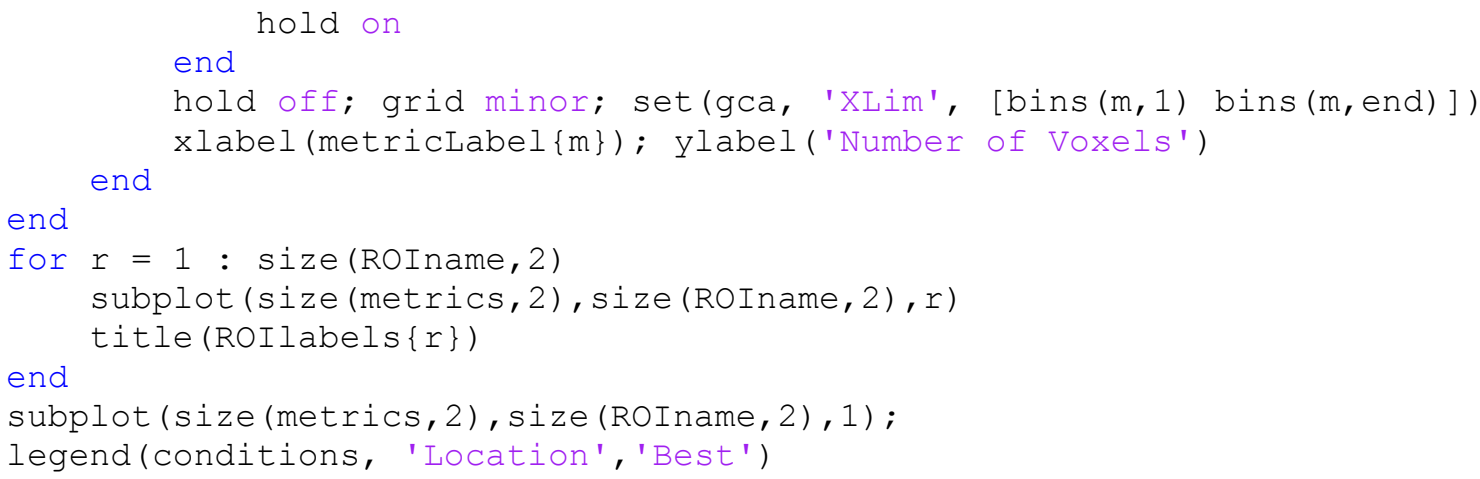

\section{Field-map default values}

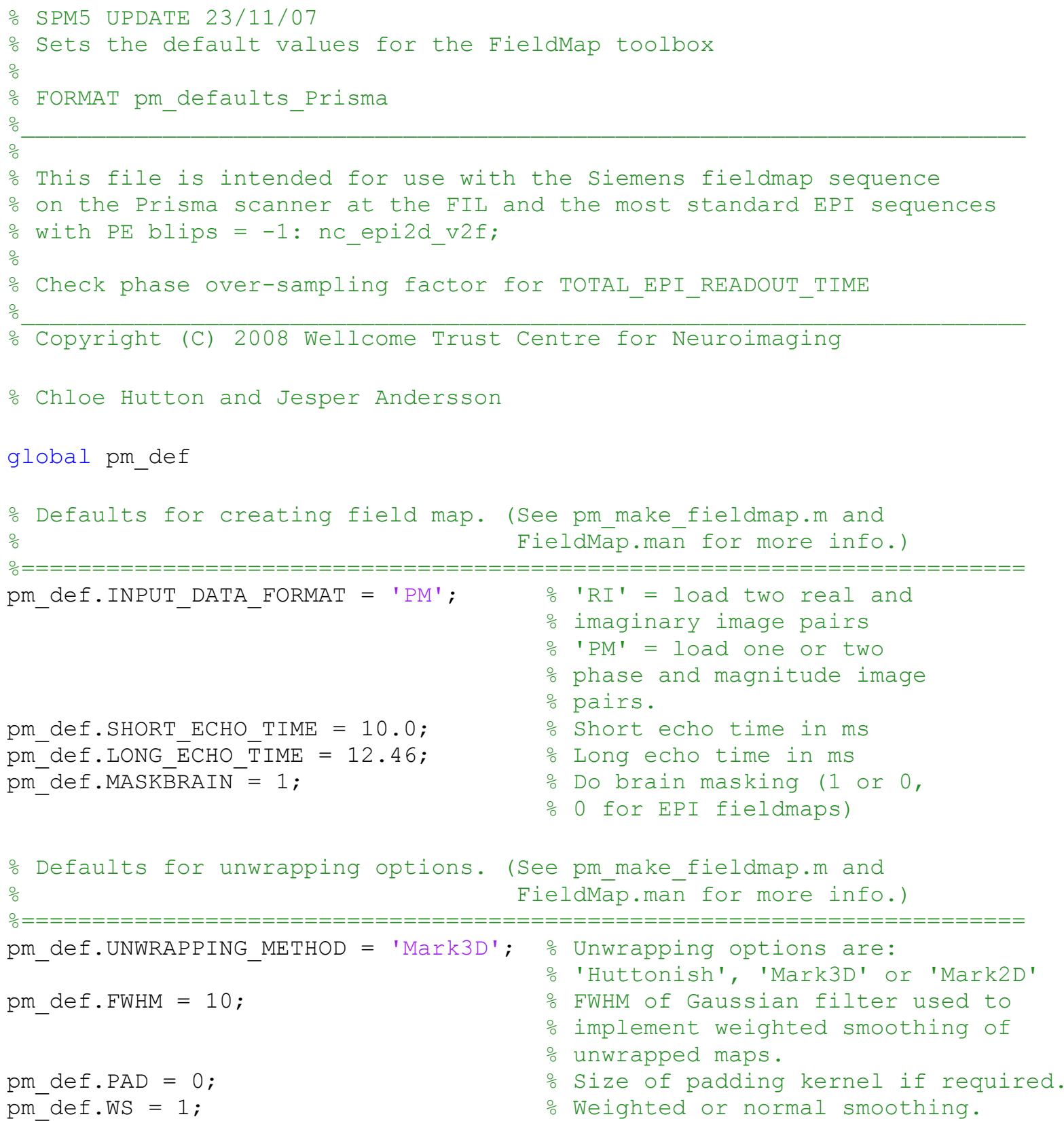




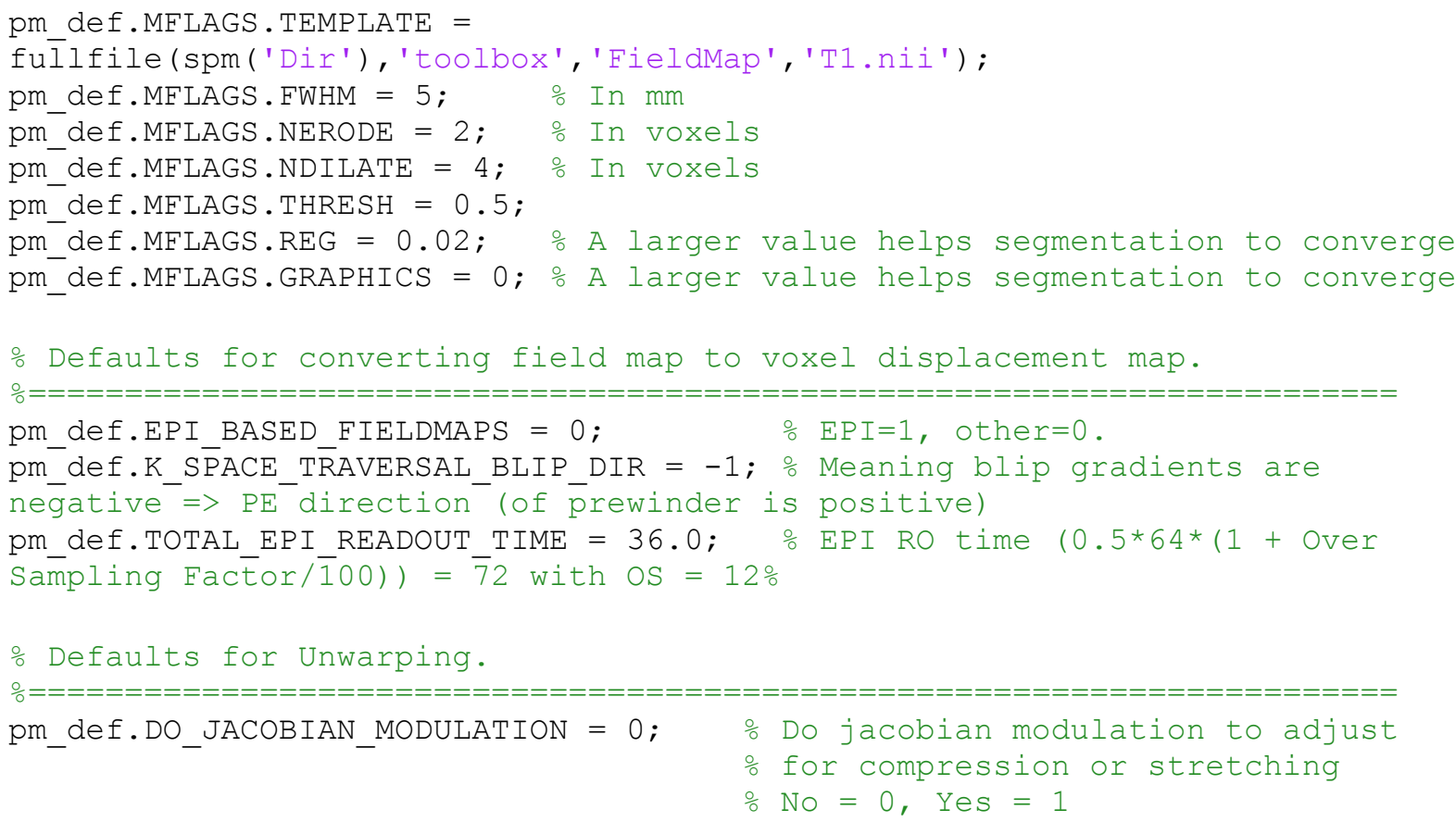

